\title{
MULTIPLE SCATTERING OF WAVES IN FRACTAL DISCRETE RANDOMLY-INHOMOGENEOUS MEDIA FROM THE POINT OF VIEW OF RADIOLOCATION OF THE SELF-SIMILAR MULTIPLE TARGETS
}

\section{Alexander A. Potapov}

Kotel'nikov Institute of Radioengineering and Electronics, Russian Academy of Science, http://cplire.ru Moscow 125009, Russian Federation

potapov@cplire.ru

Abstract: The basic elements of the classical theory of propagation and scattering of electromagnetic waves in randomly inhomogeneous media are considered. A modification of the classical FoldyTwersky theory is developed for the case of multiple scattering in fractal discrete media. Such a modification of the theory made it possible to include the values of the fractal dimension $\mathrm{D}$ and the fractal signature $D(r, t)$ of a disordered large system. The backscattering processes characteristic of radiolocation are investigated. The radar equation for a purely fractal medium is analyzed analytically. It is shown that the fractal signature can be used to study the dependence of volume scattering on distance. The present study continues the author's cycle of studies on the justification of the application of the theory of fractals, physical scaling and fractional operators in radiophysics and radiolocation, initiated by the author for the first time in the USSR in the USSR Academy of Sciences in the late 70 s of the 20th century.

Keywords: electromagnetic wave scattering, fractal randomly inhomogeneous media, fractal dimension, fractal signature, radiolocation, fractal targets

UDC 537.876.23, 530.1, 621.396.96

Bibliography - 41 references

Received 25.12.2017

RENSIT, 2018, 10(1):3-22

DOI: $10.17725 /$ rensit.2018.10.003

\section{Contents}

INTRODUCTION (3)

1. WAVES IN DISORDERED FRACTAL SYSTEMS (4)

1.1. Multiple scattering OF waVes by AN ENSEMBLE OF MOTIONLESS AND MOVING SCATTERERS: THE CLASSICAL SOLUTION OF (4)

1.2. Statistical averaging for the case of DISCRETE SCATTERERS (7)

1.3. The basic Foldy-Twersky integral EQUATION FOR THE COHERENT FIELD (8)

1.4. Twersky integral EQUATION FOR THE CORRELATION FUNCTION (9)

\subsection{The Coherent FIELd (10)}

1.6. Fractal Discrete RANDOMLY INHOMOGENEOUS MEDIA (11)

1.7. Modification of the classical FoldyTWERSKY THEORY FOR FRACTAL DISCRETE RANDOMLY INHOMOGENEOUS MEDIA (12)

2. RADAR DETECTION OF FRACTAL TARGETS (13)

2.1. The RADAR EQUATION IN TWO IDEAL PROBING CASES (14)

2.2. Fractals as models of the hierarchy of
SPACE-TIME SCALES (14)

2.3. SCATTERING OF WAVES IN A FRACTAL MEDIUM: THE FIRST NUMERICAL SIMULATION (15)

2.4. SCATtERING OF WAVES IN A FRACTAL MEDIUM: RESULTS OF NUMERICAL SIMULATION (17)

2.5. THE SCATTERING OF WAVES IN A FRACTAL MEDIUM AND THE RADIOLOCATION EQUATION (18)

3. Conclusion (19)

REFERENCES (19)

\section{INTRODUCTION}

The propagation and scattering of waves in disordered systems is considered to be one of the most difficult subjects in theoretical physics. Natural and artificial media give countless examples of diverse wave scattering on discrete particles. It is well known that when a wave propagates in a randomly inhomogeneous medium, multiple scattering must be taken into account. Phenomenological and statistical approaches are used to consider multiple scattering processes. The content of the phenomenological approach is the 
theory of radiation transfer in a scattering medium. Its apparatus is the transport equation expressing the law of conservation of radiation energy or the condition for the balance of brightness of light beams with allowance for their polarization. In the case of statistical consideration, multiple scattering of waves is based on a stochastic wave equation or from a system of such equations for which the problem of wave diffraction is posed and studied on a statistical ensemble of particles.

The theory of multiple scattering for waves in media containing random scatterers has been studied by many authors [1-9]. Since the publication of the paper [10], fractals have invariably been in the focus of the interests of various physical and technical disciplines, being suitable models for various physical phenomena [11-17]. The propagation and scattering of waves in fractal randomly inhomogeneous media is of great physical interest for radiophysics, radar, remote sensing, optics, acoustics, communication technology, biology, medicine, nanotechnology, etc. One of the original theoretical and experimental studies of scattering and diffraction on fractals was carried out in [11] (see also books [12, 13]). Although all these studies have led to the discovery of some of the fundamental physical principles, many problems associated with multiple scattering in fractal media still remain.

The theoretical basis for describing single and multiple scattering of waves by particles is formed by classical electrodynamics. In this paper, we consider in detail the problems of the general theory of multiple scattering of electromagnetic waves in fractal randomly inhomogeneous media on the basis of modifications of the FoldyTversky theory [1-3]. The main concepts of a fractal medium are introduced and the formulation of the mathematics of multiple scattering of electromagnetic waves in a fractal medium simultaneously with the physics of the scattering process is given. Modifications of the FoldyTversky integral equation for the coherent field and the Tverskii integral equation for the second moment of the field are presented.

Multiple scattering in continuous media with random fluctuations of the refractive index can also be considered. This class of problems requires special approaches to the solution, which go beyond the scope of this paper [6-8].

It should be noted that these issues were in the sphere of the author's interests even in the preparation of monographs $[12,13]$, but were not included in the general text of the books because of exceeding the set volumes. Simultaneously, these materials were included in the research reports, which were carried out by the author in the 90s. XX century. Time has shown the relevance of this topic for the problems of condensed matter physics, radiophysics and radar (see the cycle of our recent work on the dynamics of the growing fractal surface in nanotechnology and in remote sensing, in particular, for radiolocation, for example, the dynamics of the snow cover profile, etc.) [18-27].

This paper aims to generalize the study of how the problem of multiple scattering of waves on an ensemble of particles is solved and to what extent it is possible to obtain a solution of this problem for the modern theory of multiple scattering of waves in fractal discrete randomly inhomogeneous media.

\section{WAVES IN UNGROUNDED FRACTAL SYSTEMS}

\subsection{Multiple scattering OF WAVES By}

AN ENSEMBLE OF MOTIONLESS AND MOVING SCATTERERS: THE CLASSICAL SOLUTION

There are two basic approaches to the problem of wave propagation in a random cloud of scatterers - rigorous (analytical) theory and transport theory $[6,7,9]$. A rigorous theory or multiple scattering theory is based on fundamental differential equations for fields, and then statistical considerations are involved. The results of previous works were generalized by Twersky, who received a closed system of integral equations $[2,3]$. In the case of moving scatterers, the field becomes a function of time, so field correlations are observed not only in space, but also in time. Before considering the fractal medium, we give in some detail the main results of 'Twersky's classical theory, mainly based on $[2,3,7,9]$. 
RADIOELECTRONICS

Consider a cloud of $N$ particles randomly distributed in a volume $V$ with coordinates $\mathbf{r}_{1}, \mathbf{r}_{2}$, ..., $\mathbf{r}_{\mathrm{N}}$. Particles can vary in both shape and size. The scalar field $\psi^{a}$ at the point $r_{a}$ of space not occupied by particles satisfies the wave equation $\left(\nabla^{2}+k^{2}\right) \psi=0$,

where $\mathrm{k}=2 \pi / \lambda$ is the wave number in the medium surrounding the particle, $\lambda$ is the wavelength.

In equation (1), the quantity $\psi$ can describe one of the components of the electric or magnetic field. We denote $\phi_{i}^{a}$ by the incident wave at the point $\mathbf{r}_{a}$ in the absence of particles. For fields of the form $\phi_{i}^{a}$, the superscript denotes the point at which the field is considered, and the subscript indicates the origin of this field. Then the field $\psi^{a}$ at the point $\mathbf{r}_{a}$ (Fig. 1) represents the sum of the incident wave $\phi_{i}^{a}$ and the contributions $U_{s}^{a}$ from each of the $N$ particles located at the points $\mathbf{r}_{s} s=$ $1,2, \ldots, N$ :

$$
\begin{aligned}
& \psi^{a}=\phi_{i}^{a}+\sum_{s=1}^{N} U_{s}^{a}, \\
& U_{s}^{a}=u_{s}^{a} \Phi^{s} .
\end{aligned}
$$

In expression (2) $U_{s}^{a}$, a wave at the point $\mathbf{r}_{a}$, scattered by a scatterer located at the point $\mathbf{r}_{s}$. In accordance with (3) $U_{s}^{a}$, it is determined by the action of the scattering operator $u_{s}^{a}$ for the particle at the point $\mathbf{r}_{s}$ and the observation point $\mathbf{r}_{a}$ on the incident wave $\Phi^{s}$ on the particle at the point $\mathbf{r}_{s}-$ Fig. 2. The expression $u_{s}^{a} \Phi^{s}$ is the operator record of the field at the point $\mathbf{r}_{a}$, caused by the fall of the wave $\Phi^{s}$ to the scatterer located at the point $\mathbf{r}_{i}$.

In the case of a plane wave $\Phi^{s}$ propagating in the direction of the unit vector $\hat{\mathbf{1}}$, using the far-field approximation (the distance between the points $\mathbf{r}_{s}$ and $\mathbf{r}_{a}$ is large)

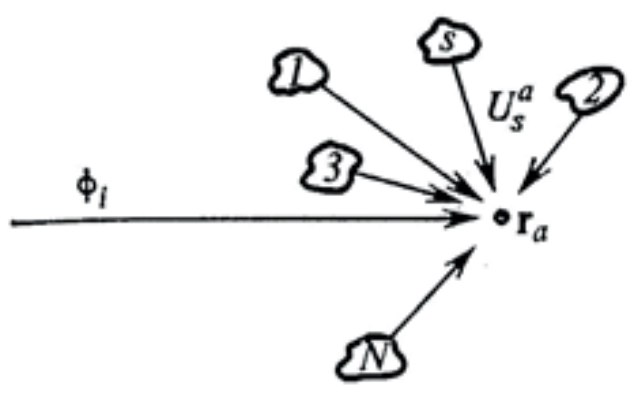

Fig. 1. The field at the point $\mathbf{r}_{a}$.

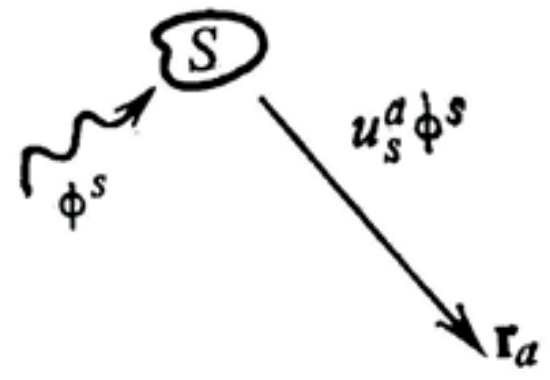

Fig. 2. Contribution from the s-th particle to the common field.

$\Phi^{\mathrm{s}}=\mathrm{e}^{\mathrm{ikr}}, \mathbf{k}=\mathbf{k} \hat{\mathbf{i}}$,

for the operator $u_{s}^{a}$, you can write

$u_{s}^{a}=f(\hat{\mathbf{O}}, \hat{\mathbf{1}}) \cdot \exp (i k r) / r$,

where $\mathbf{O}$ is the unit vector in the direction $\mathbf{r}_{a}-\mathbf{r}_{s} \mathbf{r}$ $=\left|\mathbf{r}_{a}-\mathbf{r}_{s}\right|, f(\mathbf{O}, \hat{\mathbf{1}})$ is the scattering amplitude.

The effective field $\Phi^{s}$ consists of an incident wave $\phi_{i}^{a}$ and a scattering field from all particles, with the exception of the scatterer at the point $\mathbf{r}_{\dot{s}}$. Then, as shown in Fig. 3, we have

$\Phi^{s}=\phi_{i}^{s}+\sum_{t=1, t \neq s}^{N} U_{t}^{s}$.

Equations (2) and (6) form a fundamental pair of equations.

$$
\begin{aligned}
& \psi^{a}=\phi_{i}^{a}+\sum_{s=1}^{N} u_{a}^{s} \Phi^{s}, \\
& \Phi^{s}=\phi_{i}^{s}+\sum_{t=1, t \neq s}^{N} u_{t}^{s} \Phi^{t} .
\end{aligned}
$$

Substituting $(7 b)$ in $(7 a)$ and repeating this process, we exclude the quantity $\Phi$ from these equations. Then we have:

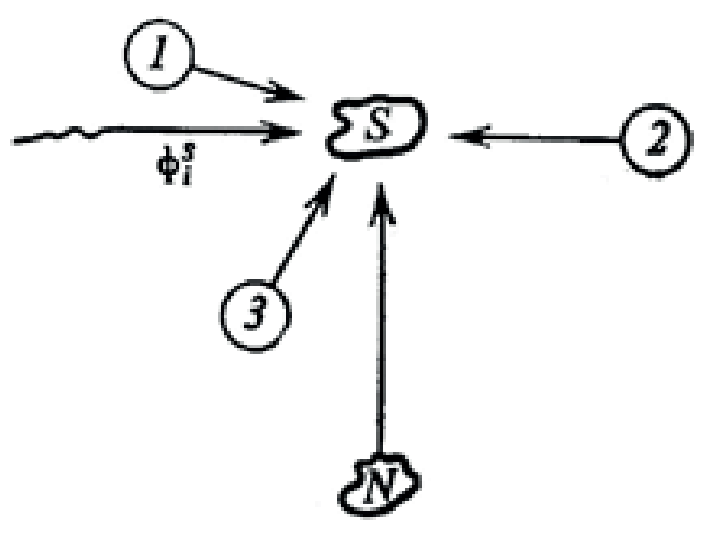

Fig. 3. An effective field for the s-th particle. 


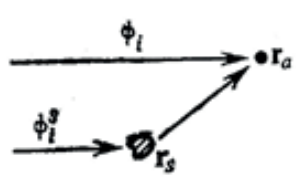

a

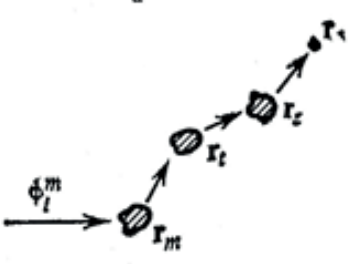

B
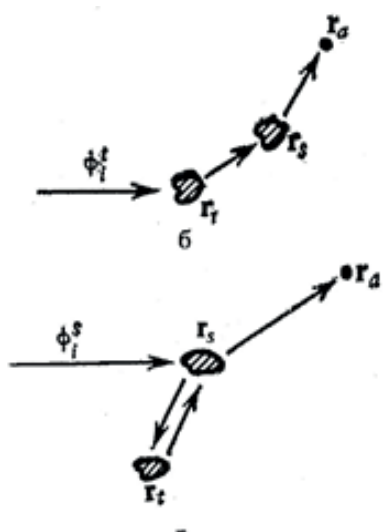

ז

Fig. 4. Schemes for single scattering (a), double scattering (b), triple scattering (c) on different particles, and the process of triple scattering when a wave of the same particle travels more than once (d).

$$
\begin{aligned}
& \psi^{a}=\phi_{i}^{a}+\sum_{s=1}^{N} u_{s}^{a}\left(\phi_{i}^{s}+\sum_{t=1, t \neq s}^{N} u_{t}^{s} \Phi^{t}\right)= \\
& \left.=\phi_{i}^{a}+\sum_{s=1}^{N} u_{s}^{a} \phi_{i}^{s}+\sum_{s=1}^{N} \sum_{t=1, t \neq s}^{N} u_{s}^{a} u_{t}^{s} \phi_{i}^{t}\right)+ \\
& +\sum_{s=1}^{N} \sum_{t=1, t \neq s}^{N} \sum_{m=1, m \neq t}^{N} u_{s}^{a} u_{t}^{s} u_{m}^{t} \phi_{i}^{m}+\ldots
\end{aligned}
$$

Now consider each term of equation (8). The first term is a falling wave $\phi_{i}^{a}$. The next term of this series

$$
\sum_{s=1}^{N} u_{s}^{a} \phi_{i}^{s}
$$

takes into account all the single scattering events (Fig. 4a), The next double sum

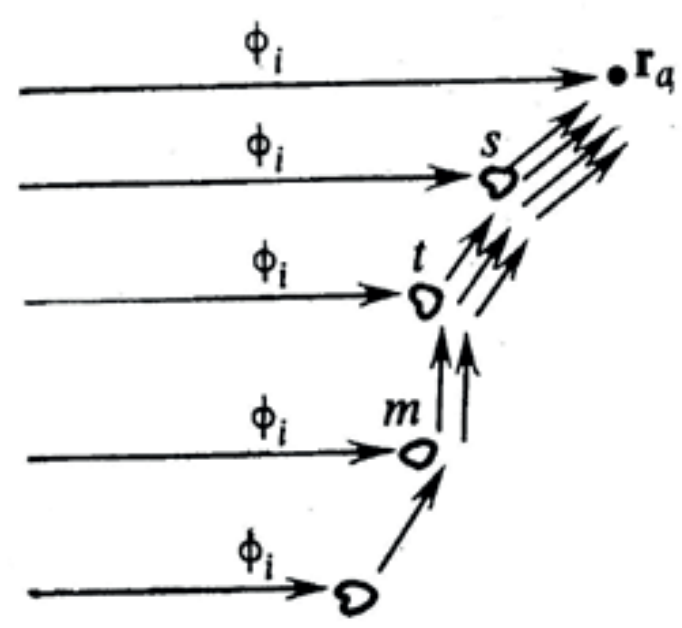

$$
\sum_{s=1}^{N} \sum_{t=1, t \neq s}^{N} u_{s}^{a} u_{t}^{s} \phi_{i}^{t}
$$

describes all the processes of double scattering (Fig. 4b).

The third amount is triple. There are no terms with $t=s$ and $m=t$, while a term with $s=m$ is present in it. This sum can be decomposed so as to single out summands with different $s, t$ and $m$ and with $s=m$ :

$\sum_{s=1}^{N} \sum_{t=1, t \neq s}^{N} \sum_{m=1, m \neq t}^{N} u_{s}^{a} u_{t}^{s} u_{m}^{t} \phi_{i}^{m}=$

$=\sum_{s=1}^{N} \sum_{t=1, t \neq s}^{N} \sum_{m=1, m \neq t, m \neq s}^{N} u_{s}^{a} u_{t}^{s} u_{m}^{t} \phi_{i}^{m}+$

$$
+\sum_{s=1} \sum_{t=1, t \neq s} u_{s}^{a} u_{t}^{s} u_{s}^{t} \phi_{i}^{s}
$$

The first triple sum from $(8 a)$ is shown in Fig. 4 ,c. In the second sum from $(8 a)$ there are only scatterers at the points $\mathbf{r}_{s}$ and $\mathbf{r}_{t}$, its graph is shown in Fig. $4 g$.

In the general case, the total field $\psi^{a}$ at the point $\mathbf{r}_{a}$, which is a superposition of the incident wave and all the multiply-scattered waves, can be divided into two parts:

1. The first part, which is described by the first sum in the expression (8a), contains all the multiply-scattered waves, taking into account the successive scattering on different scatterers. This part is illustrated by the scheme in Fig. $\mathbf{5} a$. Note that $s$ is the current index for all scatterers, so there

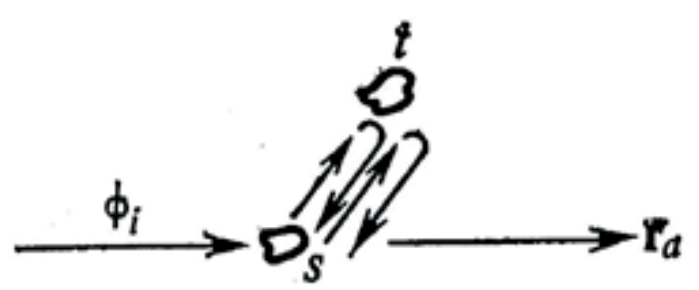

6

Fig. 5. Scheme of the paths of scattered waves passing through different scatterers (a), and a scheme of paths of scattered waves passing through the same scatterer more than once (b). 
are $N$ terms with different $s$; the index $t$ marks all the scatterers, except for $s$, that is, there is $(N-1)$ a term with different $t$. Similarly, there are $(N-2)$ terms with different $m$.

2. The second part, which is described by the second sum in the expression (8a) and corresponds to all those wave trajectories that pass through a particle more than once. This situation is shown in Fig. $5 b$.

In Twersky's theory, all the terms belonging to the first group (Fig. 5a) are taken into account and the terms belonging to the second group are neglected (Fig. 5b). Obviously, the first group describes practically all multiply scattered waves, and Twersky's theory should give excellent results if backscattering is small in comparison with scattering in other directions.

Proceeding from the above, from the mathematical point of view, Twersky's theory is based on the representation of the field in the following form:

$$
\begin{aligned}
& \left.\psi^{a}=\phi_{i}^{a}+\sum_{s=1}^{N} u_{s}^{a} \phi_{i}^{s}+\sum_{s=1}^{N} \sum_{t=1, t \neq s}^{N} u_{s}^{a} u_{t}^{s} \phi_{i}^{t}\right)+ \\
& +\sum_{s=1}^{N} \sum_{t=1, t \neq s}^{N} \sum_{m=1, m \neq t, m \neq s}^{N} u_{s}^{a} u_{t}^{s} u_{m}^{t} \phi_{i}^{m}+\ldots
\end{aligned}
$$

In Table 1 (based on the data of [7]), a comparison is made of the number of terms that are taken into account in the exact description of the multiple scattering process (8) and in the Twersky description (9). It can be seen that for large $N$, the difference between the exact description and the Twersky description is negligible.

Table1. Errors in the accurate description of multiple scattering and in Twersky.

\begin{tabular}{|c|c|c|c|}
\hline $\begin{array}{c}\text { The scattering } \\
\text { process }\end{array}$ & $\begin{array}{c}\text { Exact } \\
\text { solution } \\
{[(8)]-\mathrm{E}}\end{array}$ & $\begin{array}{c}\text { The Twersky } \\
\text { equation }[(9)]-\mathrm{T}\end{array}$ & $(\mathrm{E}-\mathrm{T}) / \mathrm{E}$ \\
\hline$\phi_{i}^{a}$ incident & 1 & 1 & 0 \\
\hline $\begin{array}{c}\text { occurring once } \\
\text { dispersion }\end{array}$ & $\mathrm{N}$ & $\mathrm{N}$ & 0 \\
\hline $\begin{array}{c}\text { Twofold } \\
\text { dispersion }\end{array}$ & $\mathrm{N}(\mathrm{N}-1)$ & $\mathrm{N}(\mathrm{N}-1)$ & 0 \\
\hline $\begin{array}{c}\text { Threefold } \\
\text { dispersion }\end{array}$ & $\mathrm{N}(\mathrm{N}-1)^{2}$ & $\mathrm{~N}(\mathrm{~N}-1)(\mathrm{N}-2)$ & $1 /(\mathrm{N}-1)$ \\
\hline $\begin{array}{c}\text { Fourfold } \\
\text { dispersion }\end{array}$ & $\mathrm{N}(\mathrm{N}-1)^{3}$ & $\mathrm{~N}(\mathrm{~N}-1)(\mathrm{N}-2)(\mathrm{N}-3)$ & $(3 \mathrm{~N}-5) /(\mathrm{N}-1)^{2}$ \\
\hline
\end{tabular}

Equation (9), which is called the Twersky expansion, is useful in understanding the physics of scattering processes, but it is inconvenient to calculate the unknown quantities. For this case, Foldy and Twersky obtained closed integral equations.

\subsection{Statistical averaging for the case of discrete scatterers}

Following [7, 9], consider the random function $f$ (field $\psi^{a}$ or product of fields), which depends on the parameters of all the scatterers. Next we consider the averaging of this function over the ensemble. Applying the probability density function $W(\underline{1}, \underline{2}, \underline{3}, \ldots, \underline{N})$, the average value of the function $f$ can be written as:

$$
\begin{aligned}
& \langle f\rangle=\iint \ldots \int f W(\underline{1}, \underline{2}, \underline{3}, \ldots, \underline{s}, \ldots, \underline{N}) d \underline{1} d \underline{2} d \underline{3} \ldots d \underline{s} \ldots d \underline{N}, \\
& d \underline{s}=d r_{s} d \varsigma_{s}=d x_{s} d y_{s} d z_{s} d \varsigma_{s},
\end{aligned}
$$

where $\mathrm{d} \mathbf{r}_{s}=d x_{s} d y_{s} d z_{s}$ is the elementary volume, $d s_{s}$ - takes into account all other characteristics of the scatterer, i.e. $d s_{s}=d$ (the shape of the $s$-th scatterer) $\times d$ (its orientation in space) $\times d$ (its size).

For a small concentration of particles and smallness of their sizes in comparison with the distance between them, all particles can be considered as point particles, and the influence of their dimensions affects only the scattering characteristics. Under this assumption we have

$$
W(\underline{1}, \underline{2}, \underline{3}, \ldots, \underline{N})=w(\underline{1}) w(\underline{2}) w(\underline{3}) \ldots w(\underline{N}) .
$$

In the case of identical statistical characteristics of particles

$$
w(\underline{s})=w\left(\mathbf{r}_{s}, \varsigma_{s}\right)
$$

integrating over all $\varsigma_{s}$, we obtain

$$
\langle f\rangle=\iint \ldots \int[f]_{\zeta} w\left(\mathbf{r}_{1}\right) w\left(\mathbf{r}_{2}\right) \ldots w\left(\mathbf{r}_{s}\right) \ldots w\left(\mathbf{r}_{N}\right) d \mathbf{r}_{1} d \mathbf{r}_{2} \ldots d \mathbf{r}_{N},
$$

where $[f]_{\varsigma}$ is the average value of $f$ corresponding to the average characteristics of the scatterer (shape, orientation, etc.).

The probability density function can be interpreted as follows:

$w\left(\mathbf{r}_{s}\right) d \mathbf{r}_{s}=$ probability of the presence of the $s$-th scatterer in an elementary volume $d \mathbf{r}_{\mathrm{s}}=$ $=\frac{\text { number of scatterers inside } d \mathbf{r}_{s}=d x_{s} d y_{s} d z_{s}}{\text { total number of scatterers in } V}=$ $=\frac{\rho\left(\mathbf{r}_{s}\right) d \mathbf{r}_{s}}{N}$, 
where $\rho\left(\mathbf{r}_{s}\right)$ is the local concentration of particles, i.e. number of scatterers in a unit volume. In this way

$$
w\left(r_{s}\right)=\frac{\rho\left(\mathbf{r}_{s}\right)}{N} .
$$

For a constant concentration of particles in a volume $V$, we also have $\rho=N / V$ and $\mathrm{w}(\mathbf{r})$ $=1 / \mathrm{V}$. In this case, the mean value (14) is given by expression

$$
\langle f\rangle=\iint \ldots \int[f]_{\varsigma} \frac{\rho\left(\mathbf{r}_{1}\right) \rho\left(\mathbf{r}_{2}\right) \ldots \rho\left(\mathbf{r}_{N}\right)}{N^{N}} d \mathbf{r}_{1} d \mathbf{r}_{2} \ldots d \mathbf{r}_{N} .
$$

If $\left[\mathbb{f}_{\varsigma}\right.$ depends only on the position of the $s$-th scatterer and does not depend on the position of the other scatterers, then []$_{\varsigma}=f\left(\mathbf{r}_{\mathrm{s}}\right)$, and we can integrate (17) over all $\mathbf{r}_{\mathrm{s}}, \ldots, \mathbf{r}_{\mathrm{s}}$, except $\mathbf{r}_{\mathrm{s}}$. As

$$
\int w\left(\mathbf{r}_{1}\right) d\left(\mathbf{r}_{1}\right)=\int \frac{\rho\left(\mathbf{r}_{1}\right) d \mathbf{r}_{1}}{N}=1
$$

we get

$$
\left\langle f\left(\mathbf{r}_{s}\right)\right\rangle=\int f\left(\mathbf{r}_{s}\right) \frac{\rho\left(\mathbf{r}_{s}\right)}{N} d \mathbf{r}_{s} .
$$

Spatial integrations are performed throughout the volume $V$.

If $[A]$ depends on the position of the two different scatterers ( $s$-th and $t$-th), then writing $[\nexists=f(\mathbf{r}, \mathbf{r})$, we get

$$
\left\langle f\left(\mathbf{r}_{s}, \mathbf{r}_{t}\right)\right\rangle=\iint f\left(\mathbf{r}_{s}, \mathbf{r}_{t}\right) \frac{\rho\left(\mathbf{r}_{s}\right) \rho\left(\mathbf{r}_{t}\right)}{N^{2}} d \mathbf{r}_{s} d \mathbf{r}_{t} .
$$

The relations (18) and (19) can easily be generalized to any number of scatterers. For large scatterer concentrations, a two-point probability distribution function must be introduced [2, 22-24].

\subsection{The basic Foldy-Twersky integral equation for a coherent field}

Suppose now that the particles filling the volume $V$ move randomly and consider the field at the interior point $\mathbf{r} \subset V$. In general, the field $\psi^{a}$ changes with time due to random time variations of the particle coordinates, although much slower than because of the time factor $\exp (-i \omega t)$. A typical measurement takes a considerable amount of time, during which an electromagnetic signal is averaged over a representative set of positions and states of particles. Consequently, it is often convenient to expand the field $\psi^{a}$ into an average (or coherent) field $\left\langle\psi^{a}\right\rangle$ and a fluctuation (or incoherent) field $\psi_{f}^{a}$.

Statistical averaging is performed on those coordinates and states of all particles that are physically realizable during the measurement time. It is very important to recognize that the coherent field $\left\langle\psi^{a}\right\rangle$ defined in this way is not a real physical field, but rather it is a purely mathematical construction. Indeed, if we restore the time harmonic factor $\exp (-i \omega t)$, which we still omit for brevity, we must conclude that the mean of the actual electric field is zero

$$
\frac{1}{T} \int_{t}^{t+T} d t^{\prime} \exp \left(-i \omega t^{\prime}\right)_{T \gg>2 \pi / \omega}=0 \text {. }
$$

On the contrary, the coherent field does not disappear, since it is defined as the time average of the electric field, which does not include the factor $\exp (-i \omega t)$. The only reason for introducing a coherent field is that it eventually appears in formulas for quantities that describe multiply scattered radiation and can in fact be measured by a suitable device. These quantities are determined in such a way that the factor $\exp (-i \omega t)$ naturally disappears when multiplied by its complex conjugate analogue.

The square of the amplitude of the mean field is a coherent intensity $\left|\left\langle\psi^{a}\right\rangle\right|^{2}$. The mean square of the amplitude of the fluctuation field is an incoherent intensity $\left\langle\left|\psi_{f}^{a}\right|^{2}\right\rangle$. The total intensity is the mean square of the total field amplitude $\left\langle\left|\psi^{a}\right|^{2}\right\rangle$ and is equal to the sum of the coherent and incoherent intensities

$$
\left\langle\left|\psi^{a}\right|^{2}\right\rangle=\left\langle\left|\left\langle\psi^{a}\right\rangle+\psi_{f}^{a}\right|^{2}\right\rangle=\left|\left\langle\psi^{a}\right\rangle\right|^{2}+\left\langle\left|\psi_{f}^{a}\right|^{2}\right\rangle .
$$

Briefly, on a qualitative level, consider, following $[2,7,9]$, the normal incidence of a plane wave on a semi-infinite region with random scatterers. The coherent intensity decreases due to scattering and absorption by law

$\mathrm{C}=$ coherent intensity $=\exp \left(-\rho \sigma_{t} z\right)$,

where $\sigma_{t}$ is the sum of the scattering and absorption cross sections.

On the other hand, the scattered power is an incoherent power, it contributes to the total 
intensity. As a result, the total intensity $T$ essentially depends only on the absorption, so that $\mathrm{T}=$ total intensity $\approx \exp \left(-\rho \sigma_{a} z\right)$.

Therefore, the incoherent intensity $I$ can be approximated by the expression

$$
I \approx \exp \left(-\rho \sigma_{a} z\right)-\exp \left(-\rho \sigma_{t} z\right)
$$

In the theory of transport, coherent and incoherent intensities correspond to attenuated incident and diffuse intensities, respectively.

Consider a coherent field, using Twersky's theory and Eq. (9). Then

$$
\begin{aligned}
& \left\langle\psi^{a}\right\rangle=\phi_{i}^{a}+\sum_{s=1}^{N}\left\langle u_{s}^{a} \phi_{i}^{s}\right\rangle+\sum_{s=1}^{N} \sum_{t=1, t \neq s}^{N}\left\langle u_{s}^{a} u_{t}^{s} \phi_{i}^{t}\right\rangle+ \\
& +\sum_{s=1}^{N} \sum_{t=1, t \neq s}^{N} \sum_{m=1, m \neq t, m \neq s}^{N}\left\langle u_{s}^{a} u_{t}^{s} u_{m}^{t} \phi_{i}^{m}\right\rangle+\ldots
\end{aligned}
$$

or using equations (17)-(19):

$\left\langle\psi^{a}\right\rangle=\phi_{i}^{a}+\sum_{s=1}^{N} \int u_{s}^{a} \phi_{i}^{s} w\left(\mathbf{r}_{s}\right) d \mathbf{r}_{s}+$

$+\sum_{s=1}^{N} \sum_{t=1, t \neq s}^{N} \iint u_{s}^{a} u_{t}^{s} \phi_{i}^{t} w\left(\mathbf{r}_{s}\right) w\left(\mathbf{r}_{t}\right) d \mathbf{r}_{s} d \mathbf{r}_{t}+$

$+\sum_{s=1}^{N} \sum_{t=1, t \neq s=1}^{N} \sum_{m=1, m \neq t, m \neq s}^{N} \iiint u_{s}^{a} u_{t}^{s} u_{m}^{t} \phi_{i}^{m} w\left(\mathbf{r}_{s}\right) w\left(\mathbf{r}_{t}\right) w\left(\mathbf{r}_{t}\right) d \mathbf{r}_{s} d \mathbf{r}_{t} d \mathbf{r}_{m}+\ldots$

Taking (16) into account, we obtain

$\left\langle\psi^{a}\right\rangle=\phi_{i}^{a}+\int u_{s}^{a} \phi_{i}^{s} \rho\left(\mathbf{r}_{s}\right) d \mathbf{r}_{s}+$

$+\frac{N(N-1)}{N^{2}} \iint u_{s}^{a} u_{t}^{s} \phi_{i}^{t} \rho\left(\mathbf{r}_{s}\right) \rho\left(\mathbf{r}_{t}\right) d \mathbf{r}_{s} d \mathbf{r}_{t}+$

$+\frac{N(N-1)(N-2)}{N^{3}} \iiint u_{s}^{a} u_{t}^{s} u_{m}^{t} \phi_{i}^{m} \rho\left(\mathbf{r}_{s}\right) \rho\left(\mathbf{r}_{t}\right) \rho\left(\mathbf{r}_{m}\right) d \mathbf{r}_{s} d \mathbf{r}_{t} d \mathbf{r}_{m}+\ldots$

In the limit $N \rightarrow \infty$ we have

$\left\langle\psi^{a}\right\rangle=\phi_{i}^{a}+\int u_{s}^{a} \phi_{i}^{s} \rho\left(\mathbf{r}_{s}\right) d \mathbf{r}_{s}+$

$+\iint u_{s}^{a} u_{t}^{s} \phi_{i}^{t} \rho\left(\mathbf{r}_{s}\right) \rho\left(\mathbf{r}_{t}\right) d \mathbf{r}_{s} d \mathbf{r}_{t}+$

$+\iiint u_{s}^{a} u_{t}^{s} u_{m}^{t} \phi_{i}^{m} \rho\left(\mathbf{r}_{s}\right) \rho\left(\mathbf{r}_{t}\right) \rho\left(\mathbf{r}_{m}\right) d \mathbf{r}_{s} d \mathbf{r}_{t} d \mathbf{r}_{m}+\ldots$

In the derivation of (27)

$\sum_{s=1}^{N}\left\langle u_{s}^{a} \phi_{i}^{s}\right\rangle=\sum_{s=1}^{N} \int\left(u_{s}^{a} \phi_{i}^{s}\right) \frac{\rho\left(\mathbf{r}_{s}\right)}{N} d \mathbf{r}_{s}=\int u_{s}^{a} \phi_{i}^{s} \rho\left(\mathbf{r}_{s}\right) d \mathbf{r}_{s}$,

$\sum_{s=1}^{N} \sum_{t=1, t \neq s}^{N} u_{s}^{a} u_{t}^{s} \phi_{i}^{t}=\sum_{s=1}^{N} \sum_{t=1, t \neq s}^{N} \iint u_{s}^{a} u_{t}^{s} \phi_{i}^{s} \frac{\rho\left(\mathbf{r}_{s}\right) \rho\left(\mathbf{r}_{t}\right)}{N^{2}} d \mathbf{r}_{s} d \mathbf{r}_{t}=$

$=\frac{(N-1)}{N} \iint u_{s}^{a} u_{t}^{s} \phi_{i}^{s} \rho\left(\mathbf{r}_{s}\right) \rho\left(\mathbf{r}_{t}\right) d \mathbf{r}_{s} d \mathbf{r}_{t}$,

which in the limit $N \rightarrow \infty$ gives

$\iint u_{s}^{a} u_{t}^{s} \phi_{i}^{s} \rho\left(\mathbf{r}_{s}\right) \rho\left(\mathbf{r}_{t}\right) d \mathbf{r}_{s} d \mathbf{r}_{t}$.

Note that the indices $s, t, \ldots$ are no longer summation indexes and are used only to denote different integration variables. Equation (27) is a complete vector version of the solution obtained by Twersky (1964) for scalar waves.

The expansion (27) is equivalent to the integral Foldy-Twersky equation

$$
\left\langle\psi^{a}\right\rangle=\phi_{i}^{a}+\int u_{s}^{a}\left\langle\psi^{s}\right\rangle \rho\left(\mathbf{r}_{s}\right) d \mathbf{r}_{s},
$$

since the integration (29) leads to (27).

The integral equation (29) is the basic equation for the coherent field in Twersky's theory. Foldy got it as a certain approximation, and Twersky established its physical meaning. The value $\left\langle\psi^{a}\right\rangle$ determined by the integral equation (29) essentially coincides with the mean value of the field $\psi^{a}$ shown in Fig. $5 a$.

1.4. Twersky integral equation for the correlation function

Let us consider the physical content of the Twersky integral equation for the intensity, which agrees with the integral Foldy-Twersky equation (29) for a coherent field [7, 9]. The Twersky integral equation can be written in the form

$$
\left\langle\psi^{a} \psi^{b *}\right\rangle=\left\langle\psi^{a}\right\rangle\left\langle\psi^{b *}\right\rangle+\int v_{s}^{a} v_{s}^{b *}\left\langle\left|\psi^{s}\right|^{2}\right\rangle \rho\left(\mathbf{r}_{s}\right) d \mathbf{r}_{s},
$$

where $v_{s}^{a}$ it satisfies the integral equation

$$
v_{s}^{a}=u_{s}^{a}+\int u_{t}^{a} v_{s}^{t} \rho\left(\mathbf{r}_{t}\right) d \mathbf{r}_{t},
$$

an asterisk indicates a transition to a complex conjugate.

The second moment of the field $\left\langle\psi^{a} \psi^{b *}\right\rangle$ is determined by a pair of integral equations (30) and (31). To explain the physical meaning of these equations, it is necessary to take the iteration of these equations. For equation (31) we have

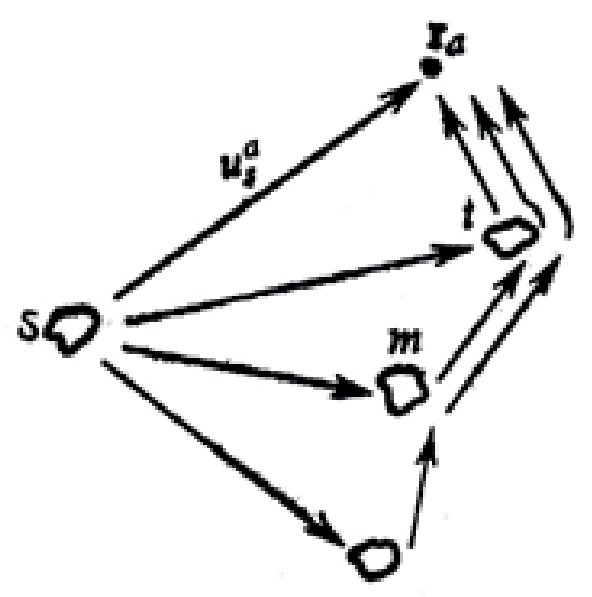

Fig. 6. Scattering processes for $v_{s}^{a}$ 


$$
\begin{aligned}
& v_{s}^{a}=u_{s}^{a}+\int u_{t}^{a} u_{s}^{t} \rho\left(\mathbf{r}_{t}\right) d \mathbf{r}_{t}+ \\
& +\int u_{t}^{a} u_{m}^{t} u_{s}^{m} \rho\left(\mathbf{r}_{t}\right) \rho\left(\mathbf{r}_{m}\right) d \mathbf{r}_{t} d \mathbf{r}_{m}+\ldots
\end{aligned}
$$

In (32), the first term $u_{s}^{a}$ describes the scattering by the scatterer $s$, which is at the point $\mathbf{r}_{a}$ (Fig. 6).

The second term in (32) in the limit $N \rightarrow \infty$ has the form

$$
\int u_{t}^{a} u_{s}^{t} \rho\left(\mathbf{r}_{t}\right) d \mathbf{r}_{t}=\sum_{t=1, t \neq s}^{N}\left\langle u_{t}^{a} u_{s}^{t}\right\rangle_{s},
$$

where the angle brackets \langle\rangle$_{s}$ mean averaging over the characteristics of the scatterer $t$ under the assumption that the parameters of the particle $s$ are fixed.

Expression (33) describes a wave scattered first by a particle $s$, and then by a particle $t$ and reaching the point $\mathbf{r}_{a}$. The third term in (32) describes the propagation of a wave from a particle $s$ to a particle $m$, then to a particle $t$, and finally to a point $\mathbf{r}_{a}$. Thus, $v_{s}^{a}$ it describes all the processes of multiple scattering from a particle $s$ to a point $a$ with the participation of different scatterers, as shown in Fig. 6.

In a similar way $[7,9]$ we integrate the integral equation (30):

$\left\langle\psi^{a} \psi^{b *}\right\rangle=\left\langle\psi^{a}\right\rangle\left\langle\psi^{b *}\right\rangle+\int v_{s}^{a} v_{s}^{b *}\left|\left\langle\psi^{s}\right\rangle\right|^{2} \rho\left(\mathbf{r}_{s}\right) d \mathbf{r}_{s}+$

$+\int v_{s}^{a} v_{s}^{b *} v_{t}^{s} v_{t}^{s *}\left|\left\langle\psi^{t}\right\rangle\right|^{2} \rho\left(\mathbf{r}_{s}\right) \rho\left(\mathbf{r}_{t}\right) d \mathbf{r}_{s} d \mathbf{r}_{t}+$

$+\int v_{s}^{a} v_{s}^{b *} v_{t}^{s} v_{t}^{s *} v_{m}^{t} v_{m}^{t *}\left|\left\langle\psi^{m}\right\rangle\right|^{2} \rho\left(\mathbf{r}_{s}\right) \rho\left(\mathbf{r}_{t}\right) \rho\left(\mathbf{r}_{m}\right) d \mathbf{r}_{s} d \mathbf{r}_{t} d \mathbf{r}_{m}+\ldots$

The first term (34), having the form $\left\langle\psi^{a}\right\rangle\left\langle\psi^{b *}\right\rangle$, is the product of the coherent field at the point $a$ by the complex conjugate coherent field at the point $b$. Since $\left\langle\psi^{a}\right\rangle$ it is the mean value of the field $\psi^{a}$, which corresponds to the sum of all the multiple scatterings shown in Fig. $5 a$, this term can be represented, as shown in Fig. 7 a.

The next member

$\int v_{s}^{a} v_{s}^{b s}\left|\left\langle\psi^{s}\right\rangle\right|^{2} \rho\left(\mathbf{r}_{s}\right) d \mathbf{r}_{s}$

represents the wave at point $a$, generated by the scattering process (Fig. 6) of the coherent field at the point $s$, and the wave at the point $b$ caused by the scattering of the complex-conjugate field $v_{s}^{b *}$. This term is shown in Fig. 7b. A similar image of the third term is shown in Fig. $7 c$.

Continuing this process, we come to the conclusion that Twersky's integral equation can be obtained by averaging the product of the fields $\psi^{a}$ and $\left\langle\psi^{b *}\right\rangle$ given by the basic scattering processes (9), illustrated in Fig. 5a. Thus, both the FoldyTwersky integral equation for the coherent field and the Twersky integral equation for the intensity take into account the same scattering processes described by the expression (9), and therefore these equations are consistent with each other.

We note that these equations correspond to the first smoothed approximation in the more rigorous Dyson and Bethe-Salpeter equations, which can be derived by means of diagram methods $[6,7,9]$.

\subsection{Coherent Field}

Consider the case of a normal incidence of a plane wave on a layer of thickness $d$ containing a large number of scatterers $[7,9]$. The incident wave propagating along the $₹$ axis is given by $\phi_{i}(r)=e^{i k z}$.

It is necessary to determine the coherent field $\langle\psi\rangle$ inside the layer, which satisfies the FoldyTwersky integral equation (29). The coherent

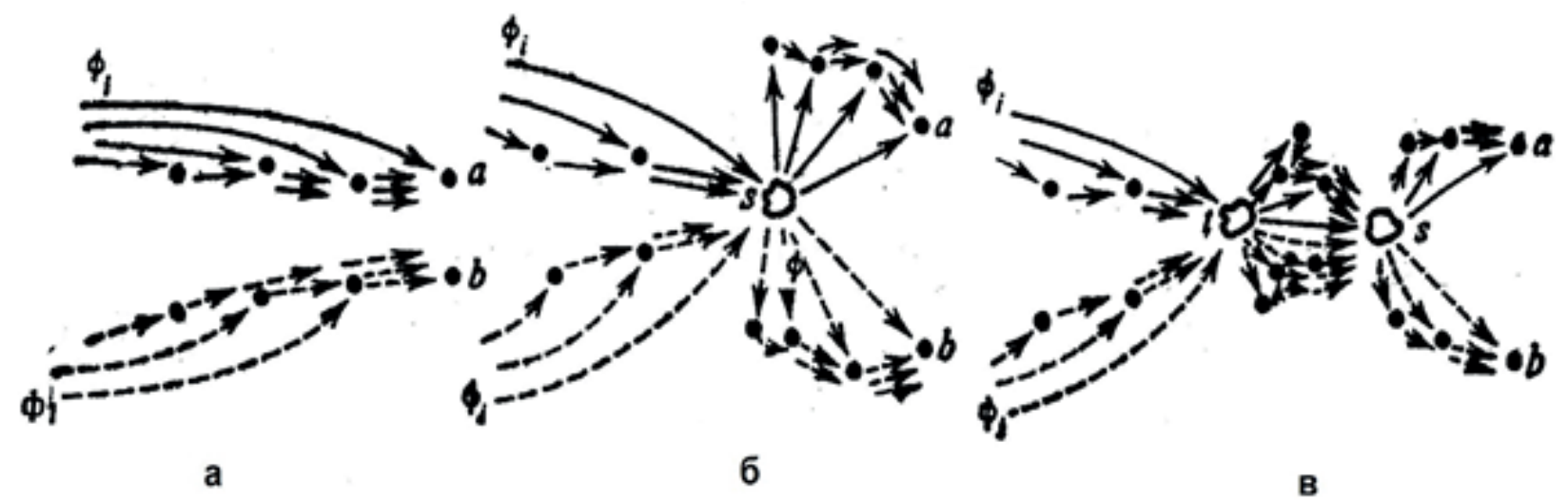

Fig. 7. Scattering processes corresponding to the first (a), the second (b) and the third (c) terms of the equation (34). 
field $\langle\psi\rangle$, like the geometry of the layer, does not depend on the coordinates $x$ and $y$, so $\langle\psi\rangle$ it must correspond to a plane wave propagating in the $+z$ direction. In the case of the location of the point $\mathbf{r}_{a}$ in the far zone with respect to the scatterer at the point $\mathbf{r}_{a}$, approximately

$$
u_{s}^{a}\left\langle\psi^{s}\right\rangle=f(\hat{\mathbf{0}}, \hat{\mathbf{i}}) \frac{\exp \left(i k\left|\mathbf{r}_{a}-\mathbf{r}_{s}\right|\right)}{\left|\mathbf{r}_{a}-\mathbf{r}_{s}\right|}\left\langle\psi^{s}\right\rangle,
$$

where $\hat{\mathbf{i}}$ is the unit vector in the direction of propagation $\left\langle\psi^{s}\right\rangle, \hat{\mathbf{0}}$ is the unit vector in the direction $\mathbf{r}_{a}-\mathbf{r}_{s}$.

Using (35) and (36), we reduce the integral equation (29) to the form

$$
\begin{aligned}
& \langle\psi(z)\rangle=e^{i k z}+ \\
& +\int_{0}^{d} d z_{s} \int_{-\infty}^{\infty} d x_{s} \int_{-\infty}^{\infty} d y_{s} f(\hat{\mathbf{0}}, \hat{\mathbf{i}}) \frac{\exp \left(i k\left|\mathbf{r}-\mathbf{r}_{s}\right|\right)}{\left|\mathbf{r}-\mathbf{r}_{s}\right|} \rho\left(z_{s}\right)\left\langle\psi\left(z_{s}\right)\right\rangle .
\end{aligned}
$$

When finding a coherent field inside the $0<z<d$ layer, we integrate the integration in (37) with respect to the variables $x_{s}$ and $y_{s}$ by the stationary phase method. Then, neglecting in the final expression the integral with a factor $f(-\hat{\mathbf{i}}, \hat{\mathbf{i}})$, which is the backscattering amplitude, the integral equation (37) takes the form

$$
\begin{aligned}
& \langle\psi(z)\rangle=e^{i k z}+ \\
& +\int_{0}^{z} d z_{s} \frac{2 \pi i}{k} \exp \left[i k\left(z-z_{s}\right)\right] f(\hat{\mathbf{i}}, \hat{\mathbf{i}}) \rho\left(z_{s}\right)\left\langle\psi\left(z_{s}\right)\right\rangle .
\end{aligned}
$$

Assuming further that the particle density is constant, we have

$$
\begin{aligned}
& \langle\psi(z)\rangle= \\
& \left.e^{i k z}\left[1+\frac{2 \pi i}{k} f(\hat{\mathbf{i}}, \hat{\mathbf{i}}) \rho \int_{0}^{z} \exp \left(-i k z_{s}\right)\right]\left\langle\psi\left(z_{s}\right)\right\rangle d z_{s}\right] .
\end{aligned}
$$

The integral equation (39) is solved exactly by using the substitution

$$
\langle\psi(z)\rangle=A e^{i K z} \text {. }
$$

As a result, we get

$$
\mathrm{A}=1, K=k+\frac{2 \pi f(\hat{\mathbf{i}}, \hat{\mathbf{i}}) \rho}{k} \text {. }
$$

Solution (40) and (41) means that when a plane wave is incident on a layer, the mean field propagates in a layer with propagation constant $K$. In the general case of an arbitrary wave incident on a layer, the mean field $\langle\psi\rangle$ can be described, assuming that it satisfies the wave equation

$$
\left(\nabla^{2}+K^{2}\right)\langle\psi(\mathbf{r})\rangle=0
$$

where $K=k+\frac{2 \pi f(\hat{\mathbf{i}}, \hat{\mathbf{i}}) \rho}{k}$.

We note [7] that the scattering amplitude $f(-\hat{\mathbf{i}}, \hat{\mathbf{i}})$ is a complex quantity, even in the case of non-absorbing scatterers. Therefore, in the course of propagation, the coherent field $\langle\psi(\mathbf{r})\rangle$ is attenuated. This type of attenuation is due to scattering and is related to the scattering cross section. For an explanation, consider the coherent intensity of a plane incident wave. In this case we have

$$
|\langle\psi(z)\rangle|^{2}=\exp \left\{-\left[\frac{4 \pi \rho}{k} \operatorname{Im} f(\hat{\mathbf{i}}, \hat{\mathbf{i}})\right] z\right\} .
$$

According to the optical theorem, which follows from the law of conservation of energy in the scattering of waves

$$
\frac{4 \pi}{k} \operatorname{Im} f(\hat{\mathbf{i}}, \hat{\mathbf{i}})=\sigma_{s}+\sigma_{a},
$$

where $\sigma$ is the scattering cross section, $\sigma$ is the absorption cross section, i.e. the energy withdrawn from the incident wave goes to scattering and absorption.

Therefore, formula (43) takes the form

$$
|\langle\psi(z)\rangle|^{2}=\exp \left[-\rho\left(\sigma_{s}+\sigma_{a}\right) z\right], 0<z<d .
$$

For a region outside the layer $z>d$, it is necessary to substitute (40) in (39) and replace the upper limit of the integral by $d$. As a result, we get

$$
\begin{aligned}
& \langle\psi(z)\rangle=\exp [i K d+i k(z-d)], \\
& |\langle\psi(z)\rangle|^{2}=\exp \left[-\rho\left(\sigma_{s}+\sigma_{a}\right) d\right], z>d .
\end{aligned}
$$

Thus, the coherent intensity is attenuated exponentially, and the attenuation constant is proportional to the scatterers density and the total cross section $\left(\sigma_{s}+\sigma_{d}\right)$.

Although this analysis refers to the case of a dip on a plane wave layer, a generalization of this approach with the aid of equation (42) is a good approximation for many practical situations.

\subsection{Fractal discrete randomly inhomogeneous media}

First, we give a brief review of the existing theoretical methods for determining the electrodynamic characteristics of a single particle. 
These quantities are also necessary for describing the scattering of waves by an individual random particle, as well as on a small group of random particles.

The scattering of light by one particle mainly depends on three key factors: the size of the scatterer (in comparison with the wavelength), its shape and the refractive index. The unlimited variability of particles in natural and anthropogenic environments is an insurmountable problem in the theoretical description of processes of propagation and scattering of waves on such particles or in clusters of such scatterers.

The case of spherically symmetric particles is an exception, since it can be easily handled using the classical Lorentz-Mie theory (very efficient and numerically exact) or one of its extensions. Most existing exact theoretical approaches fall into one of two broad categories.

The methods of differential equations give a scattered field through the solution of Maxwell's equations or a vector wave equation in the frequency or time domain, whereas the methods of integral equations are based on volume or surface integral analogs of Maxwell's equations.

Any approximate theory of wave scattering is based on a simplifying assumption, which substantially limits its range of applicability. The practical significance of approximate theories decreases as different exact methods mature and become applicable to a wider range of problems, while computers are becoming more powerful.

However, approximate theories are still a valuable source of physical understanding of the processes of scattering and absorption by nonspherical particles. In addition, it is likely that at least one approximation of geometric optics will never become obsolete, since its accuracy improves as the particle size parameter grows, whereas all the exact theoretical methods for non-spherical particles cease to be practically usable when the size parameter exceeds a certain threshold. However, this method is approximate by definition, and its range of applicability under the conditions of the parameter of the smallest size should be verified by comparing the results obtained with exact numerical solutions of the Maxwell's equations.
A review of existing theoretical and experimental methods for determining singleparticle characteristics and further references can be found in the book [28].

In this paper, we consider the problem of multiple scattering only for radar problems, we are not talking about high-resolution optics and lidar systems.

Therefore, fractality should primarily affect the spatial distribution of scattering particles, i.e. we are talking about large spatiotemporal fractal clusters [12,13]. A layer of snow can be an example of such a fractal scattering medium.

Concerning the fractal shape of an individual particle (ice crystals in clouds and snow cover), it can be seen that modern theoretical and experimental studies in optics and the IR region have shown $[9,12,13,28]$ that a rather uniform scattering amplitude prevails $f(\hat{\mathbf{0}}, \hat{\mathbf{i}})$ in comparison with other forms of particles in the entire range of scattering angles $0 \ldots 180^{\circ}$.

\subsection{Modification of the Classical Foldy- TWERSKY THEORY FOR FRACTAL DISCRETE RANDOMLY INHOMOGENEOUS MEDIA}

As is well known, the idea of fractality is based on the absence of the characteristic length, i.e. on selfsimilarity [10, 12-14]. In fact, the fractal dimension of $D$ shows how tightly the configuration of the environment or object fills the metric space in which they are located. For scattering and diffraction problems, the application of fractal ideas is widely represented in books $[12,13]$.

We now discuss some details of the modification of the classical Foldy-Twersky theory for fractal discrete randomly inhomogeneous media.

Suppose there is a fractal object of size $l_{0}$. From the definition of the fractal dimension $D$, it follows that if the length or scale varies from $l_{0}$ to $l\left(l<l_{0}\right), N$ objects containing a fraction of the fractal are obtained

$N=\left(\frac{l_{0}}{l}\right)^{D}$.

If all fractals are similar, then we have a homogeneous fractal. We denote by $E$ the Euclidean dimension, and rewrite (48) in the form 


$$
N l^{E}=\left(\frac{l_{0}}{l}\right)^{D} l^{E} .
$$

The probability density function $w(15)$ for a fractal medium can be estimated as follows. The probability of occupying a fractal of a part of space $w\left(\zeta\right.$ is its volume $N l^{\mathrm{E}}$, divided by the total volume $l_{0}^{E}$. Then

$$
w(l)=\frac{N l^{E}}{l_{0}^{E}}=\left(\frac{l}{l_{0}}\right)^{E-D} .
$$

When the Foldy-Twersky theory is modified for a fractal scattering medium, in the first approximation, the expression (50) must be taken into account in the integral equations for the coherent field (26) and (27). Then

$$
\begin{aligned}
& \left\langle\psi^{a}\right\rangle=\phi_{i}^{a}+\int u_{s}^{a} \phi_{i}^{s} w\left(\mathbf{r}_{s}\right) d \mathbf{r}_{s}+ \\
& +\iint u_{s}^{a} u_{t}^{s} \phi_{i}^{t} w\left(\mathbf{r}_{s}\right) w\left(\mathbf{r}_{t}\right) d \mathbf{r}_{s} d \mathbf{r}_{t}+ \\
& +\iiint u_{s}^{a} u_{t}^{s} u_{m}^{t} \phi_{i}^{m} w\left(\mathbf{r}_{s}\right) w\left(\mathbf{r}_{t}\right) w\left(\mathbf{r}_{m}\right) d \mathbf{r}_{s} d \mathbf{r}_{t} d \mathbf{r}_{m}+\ldots
\end{aligned}
$$

Similarly, you can perform the same mathematical operations for a cascade of fractals nested into each other, or for a chain of fractals. These types of complex fractal and multifractal clusters are widely distributed in nature and nanotechnology [12-15].

Equations (30) and (34) of the second moment of the field $\left\langle\psi^{a} \psi^{b *}\right\rangle$ for a fractal scattering medium take the form respectively

$$
\begin{aligned}
& \left\langle\psi^{a} \psi^{b *}\right\rangle=\left\langle\psi^{a}\right\rangle\left\langle\psi^{b *}\right\rangle+\int v_{s}^{a} v_{s}^{b^{*}}\left\langle\left|\psi^{s}\right|^{2}\right\rangle w\left(\mathbf{r}_{s}\right) d \mathbf{r}_{s}, \\
& \left\langle\psi^{a} \psi^{b *}\right\rangle=\left\langle\psi^{a}\right\rangle\left\langle\psi^{b *}\right\rangle+\int v_{s}^{a} v_{s}^{b *}\left|\left\langle\psi^{s}\right\rangle\right|^{2} w\left(\mathbf{r}_{s}\right) d \mathbf{r}_{s}+ \\
& +\int v_{s}^{a} b_{s}^{b *} v_{t}^{s} v_{t}^{s *}\left|\left\langle\psi^{t}\right\rangle\right|^{2} w\left(\mathbf{r}_{s}\right) w\left(\mathbf{r}_{t}\right) d \mathbf{r}_{s} d \mathbf{r}_{t}+ \\
& +\int v_{s}^{a} v_{s}^{b s} v_{t}^{s} v_{t}^{s s^{s}} v_{m}^{t} v_{m}^{t *}\left|\left\langle\psi^{m}\right\rangle\right|^{2} w\left(\mathbf{r}_{s}\right) w\left(\mathbf{r}_{t}\right) w\left(\mathbf{r}_{m}\right) d \mathbf{r}_{s} d \mathbf{r}_{t} d \mathbf{r}_{m}+\ldots
\end{aligned}
$$

where

$$
v_{s}^{a}=u_{s}^{a}+\int u_{t}^{a} v_{s}^{t} w\left(\mathbf{r}_{t}\right) d \mathbf{r}_{t} .
$$

We calculate the coherent field $\langle\psi\rangle$ for a normal incidence of a plane wave on a fractal layer of thickness $d$ containing a large number of scatterers. The incident field has the form of a plane wave (35) propagating along the zaxis. Then:

$$
\begin{aligned}
& \langle\psi(z)\rangle=e^{i k z}+ \\
& \int_{0}^{d} d z_{s} \int_{-\infty}^{\infty} d x_{s} \int_{-\infty}^{\infty} d y_{s} w\left(z_{s}\right) f(\hat{\mathbf{0}}, \hat{\mathbf{i}}) \frac{\exp \left(i k \mid \mathbf{r}-\mathbf{r}_{s}\right)}{\left|\mathbf{r}-\mathbf{r}_{s}\right|}\left\langle\psi\left(z_{s}\right)\right\rangle .
\end{aligned}
$$

Using the above procedure (see 1.5 of this paper), it can be obtained that the average field $\langle\psi\rangle$ in a fractal scattering medium satisfies the wave equation

$$
\left(\nabla^{2}+K^{2}\right)\langle\psi(\mathbf{r})\rangle=0,
$$

where

$$
K=k+\frac{2 \pi f(\hat{\mathbf{i}}, \hat{\mathbf{i}}) w(\mathbf{r})}{k} .
$$

The integral equation (29) in accordance with (37) for the fractal layer has the form

$$
\begin{aligned}
& \langle\psi(z)\rangle=e^{i k z}+ \\
& +\int_{0}^{d} d z_{s} \int_{-\infty}^{\infty} d x_{s} \int_{-\infty}^{\infty} d y_{s} f(\hat{\mathbf{0}}, \hat{\mathbf{i}}) \frac{\exp \left(i k\left|\mathbf{r}-\mathbf{r}_{s}\right|\right)}{\left|\mathbf{r}-\mathbf{r}_{s}\right|} w\left(z_{s}\right)\left\langle\psi\left(z_{s}\right)\right\rangle .
\end{aligned}
$$

From the solution of the wave equation (55), the coherent intensity of a plane incident wave is

$$
|\langle\psi(z)\rangle|^{2}=\exp \left\{-\left[\frac{4 \pi w(z)}{k} \operatorname{Im} f(\hat{\mathbf{i}}, \hat{\mathbf{i}})\right] z\right\} .
$$

For a fractal layer of thickness $d$, the coherent intensity of a plane incident wave, taking into account the optical theorem (44) and equations (45) and (47), has the form

$$
\begin{aligned}
& |\langle\psi(z)\rangle|^{2}=\exp \left[-w(r)\left(\sigma_{s}+\sigma_{a}\right) d\right], \text { for } \mathrm{z}>\mathrm{d} \text {. } \\
& |\langle\psi(z)\rangle|^{2}=\exp \left[-w(r) r\left(\sigma_{s}+\sigma_{a}\right) z\right], \text { for } 0<\mathrm{z}<\mathrm{d} \text {. }
\end{aligned}
$$

Thus, on the basis of the modification of the classical Foldy-Twersky theory, a general theory developed for multiple scattering of waves in fractal randomly inhomogeneous media is obtained.

\section{RADAR LOCATION OF FRACTAL TARGETS}

The modified Foldy-Twersky method for multiple scattering of waves in fractal discrete randomly inhomogeneous media has a very general character and allows one to consider in a unified way a large number of wave phenomena generated by the theory of fractals and the practice of their application. The constructed modification of the multiple scattering theory allowed us to include the values of the fractal dimension $D$ and the fractal signature $D(\mathbf{r}, t)$ of the disordered large system, 
which allows us to justify solutions for anisotropic disordered large fractal systems.

In the present work, backscattering processes characteristic of radiolocation are investigated. In the radar sounding of a target in the atmosphere, the dependence of the power of the received signal on the distance $r$ is well known in at least two ideal cases. The power of the signal from the point target falls as an inverse fourth degree from the observation range and as an inverse square from the range for a homogeneous medium. Inhomogeneous natural environments, such as the average atmosphere, have intermittent or heterogeneous structures that do not correspond to any of these cases. Thin, vertically stratified undulating turbulence layers are often observed in the entire middle atmosphere. Therefore, the validity of the exact dependence of signal power on $r^{4}$ or $r^{2}$ in radar experiments of the middle atmosphere remains questionable [29].

Many natural objects that have structural elements in the hierarchy of space-time scales challenge the smooth functions. Their structure can be convincingly represented with the help of fractals $[12,13]$. Therefore, for fractal modeling, intermediate cases of inhomogeneous or randomly inhomogeneous media are of interest. Such real fractal models, which include stratification effects, can be useful in radiophysical and radar research.

The dependence of the received power on the fractal signature of the volume or target was studied for the radar case. It is shown that the fractal signature can be used to study the dependence of volume scattering on distance. Theoretical studies are consistent with previously published results of foreign authors.

\subsection{The RADAR EQUation IN TWO IDEAL PROBING CASES}

The change in the power of the scattered signal $P$. in two ideal cases is given by the radar equation. Consider a monostatic radar experiment at wavelength $\lambda_{0}$ (frequency $f_{0}$ ), antenna aperture size $d$, antenna gain $G=4 \pi A / \lambda^{2}$, where $A$ is the effective area of the antenna. The antenna beamwidth $\theta_{a}=\alpha \lambda_{0} / d$, coefficient $\alpha \approx 1$. We assume that the target is in the far zone.
First, we consider a point target with an effective cross section $\sigma$ located at a distance $r$. The received signal power is determined by the radar equation

$$
P_{s}=\frac{P_{t} G}{4 \pi r^{2}} \times \sigma \times \frac{A}{4 \pi r^{2}} \times L=\frac{P_{t} A^{2} L \sigma}{4 \pi \lambda_{0}^{2} r^{4}},
$$

where $P_{t}$ is the transmitter power, $L$ is the coefficient that takes into account all the losses. It is seen that $P_{t}$ falls off with a range of $r$ as $r^{4}$.

Next, we consider a homogeneous ensemble of a set of pointwise randomly distributed in space. If we assume that the point targets are statistically independent, then the power of the received signal $P_{s}$ is obtained by summing the energy contributions from all point targets in the ensemble. The efficiency of scattering by an ensemble of electromagnetic waves is determined by the cross section $\sigma_{s}$ per unit volume. The region of the medium that contributes to $P$ is at a distance $r$ and is determined by the beamwidth $\theta$ and the radial resolution $\Delta r$. Thus, the effective volume contributing to $P_{s}$ is $V=r^{2} \delta \Omega \Delta r$, where $\delta \Omega=\theta_{a}^{2} / 4$ is the solid angle of the ray. Then the radar equation becomes

$P_{s}=\frac{P_{t} G}{4 \pi r^{2}} \times V \sigma_{v} \times \frac{A}{4 \pi r^{2}} \times L=\frac{P_{t} A \alpha^{2} L}{64 r^{2}} \times \Delta r \sigma_{v}$.

The dependence $P$ as the inverse square of the distance $r$ is determined by the fact that the volume $V$ increases as $r^{2}$, and from equation (61) it follows that the energy contribution of each point target to the volume $V$ decreases as $r^{4}$. In addition, $P_{s}$ does not depend on the radar wavelength $\lambda_{0}$, except through $\sigma_{v}$.

Another case occurs when probing the earth's surface at low slip angles $\vartheta$. In this case, the area of the ground distributed target illuminated by the antenna beam increases linearly with the range $r$. Assuming the statistical independence of the elementary scatterers of the distributed target, we obtain that the power $P$ decreases with increasing distance as $r^{3}$.

The assumption of statistical independence of point targets in a flat region or volume usually requires caution. So in [29, p. 192] there is the assertion that "... the problems associated with the influence of local objects are quite complex 
and have not yet been fully resolved, and only relatively recently meteorological radar specialists have begun to pay attention to this field of

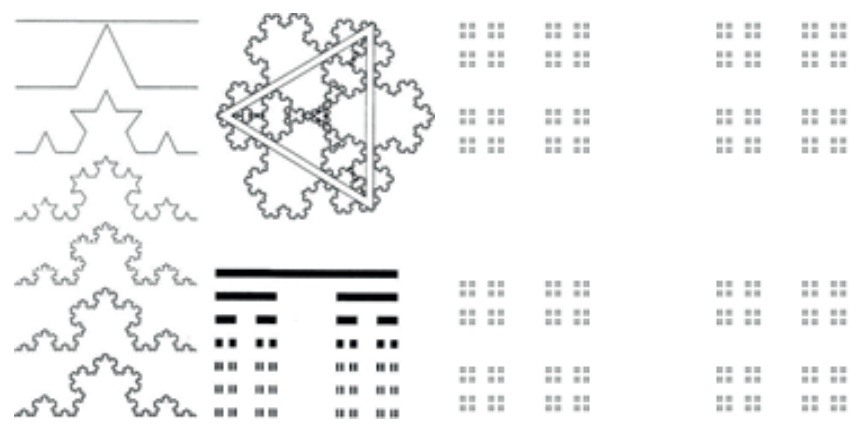

Fig. 8.1. Examples of fractals: the Koch curve and its construction (left), the Koch islands (above in the center), the dust of Cantor (at the bottom and right of 2D on an enlarged scale).

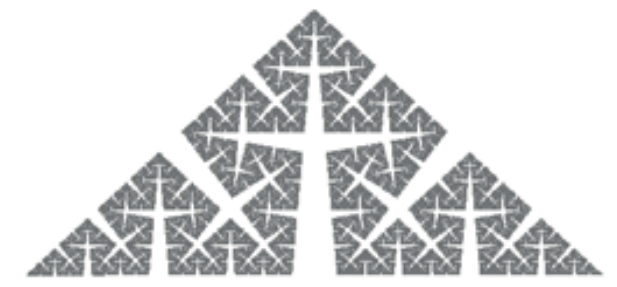

Fig. 8.2. The Cesaro fractal is a variant of the Koch curve with an angle between $60^{\circ}$ and $90^{\circ}$ (here $85^{\circ}$ ), $1 \mathrm{D}$.

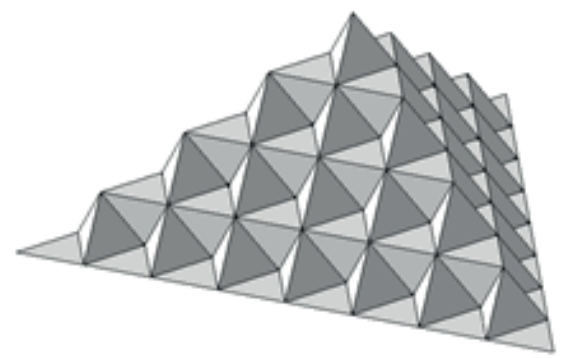

Fig. 8.3. Variants: Koch surface (extending the Koch curve to $2 D)$.



Fig. 8.4. Variants: The Koch curve is 3D. Three-dimensional fractal from the curves of Koch. The form can be considered as a three-dimensional extension of the curve in the same sense that the Sierpinski pyramid and the Menger sponge can be regarded as an extension of the Sierpinski triangle and Sierpinsky napkin research." The exact power-law dependence of $P$ on the distance $r$ is a function of how these inhomogeneities or irregularities fill the scattering region. This dependence varies from $r^{4}$ for a point target to $r^{2}$ for a homogeneous medium that completely fills the entire scattering region of $V$.

Intermediate cases are very important when probing real randomly inhomogeneous media, but they do not lend themselves to analysis. The author knows only one work on computer modeling of wave scattering by media with partial filling of the sounding space on the basis of fractal approximation [30] (see also [12, 13]). In Sections 2.3 and 2.4, we present the main results of this work for further research.

\subsection{Fractals as models of the hierarchy of SPACE-TIME SCALES}

The theory of fractals was first developed and presented to the world by the great mathematician B. Mandelbrot (1924-2010). This happened in 1975. The global introduction of fractal mathematics and physics into radiophysics, radar, and a wide range of other scientific areas has been carried out by the author of this work since 1979 [12, 13]. In Fig. 8 shows selected examples of fractal sets.

A digital simulation of planar fractal targets was carried out in [30]. The region of space is divided into smaller cells, eventually reducing to isotropic point targets. The process begins with a unit square (Euclidean dimension $E=2$ ). Then the square is divided into $n^{2}$ equal subcells with side $n^{-1}$, and this process is repeated. The dimension $E=2$ of Euclidean space is preserved, since $E=2=\log \left(n^{2}\right) / \log (n)$.

Suppose now that in the division stage only $p \geq n^{-2}$ sub-cells are filled, and the process recursively continues for these filled sub-cells. After the iteration cycle, the unit square is filled with a random pattern of points of finite dimensions. The fractal dimension $D$ of this pattern is not more than $E$, but it can be close to 0 . At each iteration the number of subcells covered by the pattern is $p n^{2}$, and their side is reduced by the factor $n$. The fractal dimension of $D$ can now be defined as $\{2+\log (p) / \log (n)\}$. The incremental factor is actually negative or zero, since $p \leq 1$. 

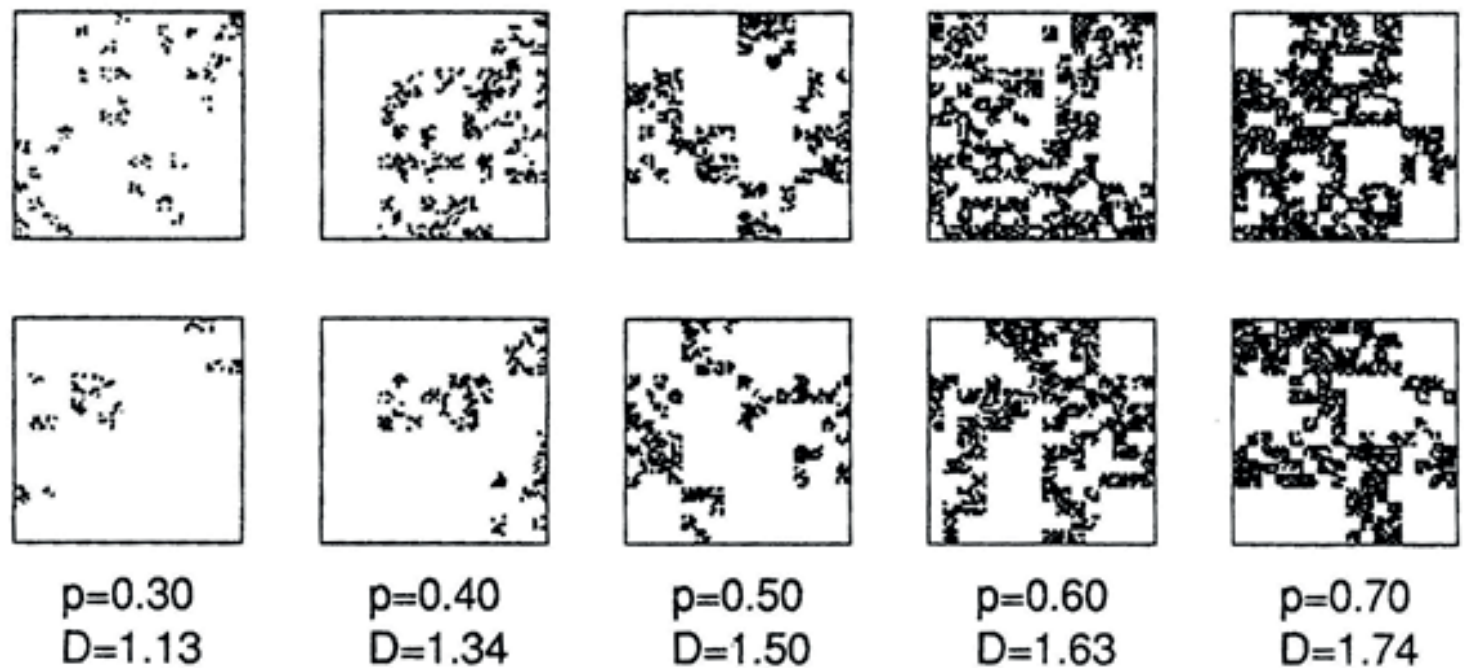

$D=1.34$
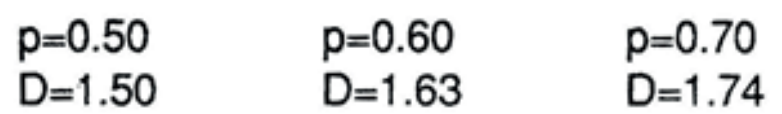

Fig. 9. Random dot patterns on the grid $256 \times 256$. At each stage of division, the cell is divided into $4 \times 4=16$ sub-cells, which are then selected with probability $p>4-2$. The steps are repeated four times because of the final size of the pixels. The parameters $p$ and $D$ increase from left to right. For each case, two different implementations are shown. Such patterns model specific implementations of point targets in a distributed random environment. The fractal dimension D, controlled by the probability p, determines the degree to which the medium fills the plane [30].

The fraction $p$ can also be considered as probability. The resulting pattern is really random and statistically self-similar with increasing. The degree at which a random dot pattern fills a unit square can be controlled by choosing the value of $p$.

When $p=n^{-2}$, the value of $D$ becomes zero. The extension to $E=3$ is obvious with $D=\log \left(p n^{3}\right)$ / $\log (n)$. Examples of realizations of random points on a plane are shown in Fig. 9.

These points can be considered as random point radar targets. Such examples serve as the basis for the numerical experiments described below.

\subsection{SCATTERING OF WAVES IN A FRACTAL MEDIUM:} THE FIRST NUMERICAL SIMULATION

In [30], the radar scattering process was numerically simulated to study the variations in the power of the received signal when the range to the target, the length of the probing wave, and the nature of the spatial target itself were changed.

Each point target is assigned the value of the effective scattering cross section. The point target $s_{\mathrm{i}}$ at the point $r_{\mathrm{i}}$ is illuminated by a uniform beam with respect to $\theta$. The scattering field $E_{i}$ on the receiving antenna, under the assumption of the far zone, varies with distance $r_{\mathrm{i}}$ to the point target as $\left(r_{\mathrm{i}}\right)^{-2}$, and its phase is $-4 \pi r_{\mathrm{i}} / \lambda_{0}$. The complex signal $v_{\mathrm{i}}$ at the output of the receiver is linearly connected with $E_{i}$. The signal power $P_{s}$ from the ensemble of point targets is determined from the accumulated complex voltage $v=\Sigma_{i} \cdot v_{i}$, like $P_{s}=v v^{*}$.

It should be noted that this method of determining $P_{s}$ gives the "true" signal power for any arbitrary target structure. It does not presuppose the statistical independence of point targets, and all phase factors are inherently included in the calculations.

In real modeling, to remove computational constraints, only planar goals that are twodimensional ensembles of point targets were considered (Fig. 9). This restricts us to the values of $D \leq 2$ inside a Euclidean space of dimension $E=3$.

The orientation of the planar target relative to the radar beam is shown in Fig. 10. The radar beam is directed at an angle $\theta$ from the zenith. The flat target is oriented vertically, in the plane determined by the zenith and the axis of the radar beam. Only this part of the planar target (indicated by the hatching in Fig. 10), which intersects with the radar volume at a distance $r$, forms a signal obtained from this range range. A flat target is formed by a fractal implementation of points, such as those shown in Fig. 9. 


\section{RADIOELECTRONICS}

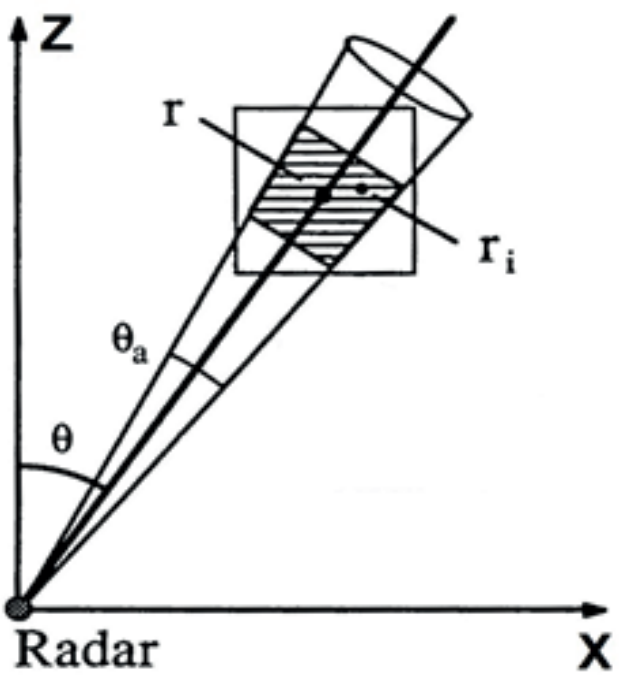

Fig. 10. Geometry of the sounding of a fractal target.

Fractal implementations of planar targets with $D \leq 2$ are generated by first dividing the area of $4096 \times 4096$ points into $164 \times 4$ cells. Each sub-cell is likewise subdivided iteratively.

The total number of iterations in this division is six $\left(4096=4^{6}\right)$. In each iteration, the subcell is included with probability $p$. Typical realizations of random points on the plane are shown in Fig. 9. It should be noted that the subcells have different horizontal and vertical boundaries, as an artifact of a simple fractal model used here. Full modeling of the volume target with $4096^{3}$ or $\sim 69$ billion points is clearly inadvisable for computational reasons.

From a physical point of view, a linear dimension of $0.1 \mathrm{~m}$ is associated with each point target. The linear dimension of the scattering region is $\sim 0.4 \mathrm{~km}$. To simplify the calculations, the beam width and the radial resolution are kept constant, namely, $\theta_{a}=0.9^{\circ}$ and $\Delta r=0.32 \mathrm{~km}$. Five values were used for each of the following parameters: nominal range $r$ from 5 to $20 \mathrm{~km}$, zenith angle $\theta$ from 0 to $20^{\circ}$ and wavelength $\lambda_{0}$ from 3 to $1 \mathrm{~m}$. The probability parameter $p$, which controls fractal dimension $D$, varied from 0.3 to 0.7 with a step of 0.1 . For each of the 625 individual cases, the $P_{s}$ value was averaged over 20 different implementations. Scaling or the scaling law for the dependence of $P_{s}$ on any parameter, for example, range, is then obtained by regression analysis. Further, only the dependence of the signal power $P$ on the range $r$ is discussed.
MULTIPLE SCATTERING OF WAVES IN FRACTAL 17 DISCRETE RANDOMLY-INHOMOGENEOUS MEDIA...

The values of the parameters are typical for typical experimental radars of the middle atmosphere [29]. On some radars that use large antennas, near-field effects are significant. They are easy to incorporate and will be of interest to our future work, since the radar equations given above are valid only for the far zone.

\subsection{SCATTERING OF WAVES IN A FRACTAL MEDIUM: RESULTS OF NUMERICAL SIMULATION}

To determine the power of the scattered signal from the range, we determine this ratio in the form of a power law [12, 13, 30]:

$$
P_{s}=r^{-\beta} \text {. }
$$

The index $\beta$ is equal to 4.0 for a point target and 2.0 for a target that spatially fills the beam, that is, $D=3$.

The index $\beta$ was obtained in [30] by linear regression of $\log (P)$ by $\log (r)$ in the numerical experiment described above for five different values of $D$ between 1.13 and 1.74 at a wavelength $\lambda_{0}=2.5 \mathrm{~m}$ and a probe angle $\theta=10^{\circ}$. In Fig. 11 shows the change in the parameter $\beta$ from the

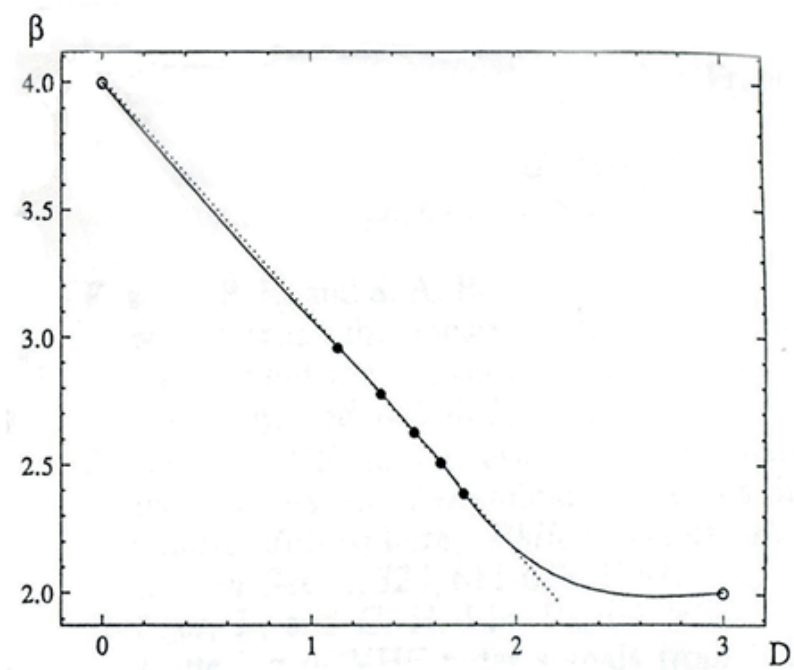

Fig. 11. Variation of the parameter $\beta$ as a function of the fractal dimension $D$ of the scattering medium. The extremal cases of a point target $(D=0)$ and volume scattering by a homogeneous random medium $(D=3)$ are shown by light circles. Black circles are average values for more than 20 realizations in a numerical experiment. The dashed line is the method of least squares. The solid line is a cubic spline passing through all seven points. The parameter $\beta$ first falls linearly with increasing $D$, but slows down near $D \sim 2$ in the case of volume scattering: $\lambda_{0}=2.5 \mathrm{~m}, \theta=10^{\circ}$. 
value of $D$ for five values of the fractal dimension D.

The extremal cases for $D=0$ and $D=3$ in Fig. 11 are also identified. Linear regressions by the method of least squares, corresponding to five calculated points, and a cubic spline corresponding to all seven points are shown. For planar targets with $D \sim 1.9, \beta \sim 2.22$ is obtained. Consequently, the volume case $(\beta=2)$ is high enough for purposes that only seek to fill the plane.

The fit of the splines shows that for $D \sim 2.2$ the bulk scattering limit at $\beta=2.0$ is kept accurate to $5 \%$ and this limit is well achieved if $D>2.4$.

As noted in [30], the above result does not necessarily hold for all orientations of the planar target. For a planar target oriented perpendicular to the beam axis and for all purposes near the vertical drop, signals from point targets in the Fresnel zone are formed coherently. These coherent reflections observed in many radar experiments [31-33] have also been observed in numerical experiments and are discussed below. Coherent reflections are produced by the boundaries of the subcell, at almost perpendicular incidence.

It is interesting to think how the results shown in Fig. 11, refer to the range $\left(r^{-3}\right)$ of the terrestrial interference power $(P)$ for radars, which was briefly discussed in Section 2.1. This situation corresponds to an almost horizontal antenna beam $\left(\theta \sim 90^{\circ}\right)$ and a horizontal flat target that contains statistically independent elements. The orientation of this planar target, however, is orthogonal to that shown in Fig. 10. It can be considered as a collection of many statistically independent linear goals, all of which are parallel to the ray. Each of these linear goals corresponds to the fractal dimension $D=1$ in Fig. 11. From the assumed statistical independence of these linear targets, the exponent of the power law $P$ should be the same as for $D=1$. The actual value of this exponent $\beta$ is $~ 3.07$ in Fig. 11. The mismatch of 0.07 in the indicator may be due to some two-point correlations within the sub-cell, contrary to the assumed statistical independence between point targets, and the possible angular $(\theta)$ dependence in numerical experiments [30].
The simple fractal model of the random medium presented in [30] is still far from realistic, since it does not include the spatial inhomogeneity manifested in stratified turbulence layers, usually observed in the atmosphere [34, 35]. Improved fractal models, for example, those that use an anisotropic cascade [12, 13, 36, 37], successfully represent such layers and can be used in the future.

\subsection{SCATTERING OF WAVES IN A FRACTAL MEDIUM AND THE RADIOLOCATION EQUATION}

The theory of multiple scattering of electromagnetic waves in fractal discrete randomly inhomogeneous media is presented by the author in $[12,13]$. Here we show how to use the results obtained in the analysis of radar signals when the scattering volume depends on the fractal dimension $D$ or the fractal signature $D(\mathbf{r}, t)$. In accordance with the theory of wave scattering, it is possible to determine the transverse scattering profile $\Sigma_{s}$ and the transverse absorption profile $\Sigma_{a}$ (which can be measured experimentally) in the form

$$
\sum_{s}=w_{s} \sigma_{s}, \quad \sum_{a}=w_{a} \sigma_{a},
$$

where $w_{s}$ is the relative volume of the fractal introduced of (50).

Correspondingly, the field moments for the fractal medium are equal (51-53).

We calculate the value of the backscattered signal from the fractal medium (Fig. 10) using the classical radar equation. The power of the received signal is determined by the radar equation

$$
P_{s}=\iint \frac{P_{t}}{4 \pi r_{1}^{2}} \sigma_{s} \frac{1}{4 \pi r_{2}^{2}} e^{-2 i k\left(r_{1}^{2}-r_{2}^{2}\right)} d r_{1} d r_{2}
$$

Taking into account (64)

$$
P_{s}=\frac{P_{t} \sum_{s}}{4 \pi^{2}} \iint \frac{1}{4 \pi r_{1}^{2} r_{2}^{2} w_{s}} e^{-2 i k\left(r_{1}^{2}-r_{2}^{2}\right)} d r_{1} d r_{2} \text {. }
$$

Using the formula (50) for the relative volume of the fractal and the results of [37], we have

$$
\begin{aligned}
& P_{s}=\frac{A P_{t} \sum_{s}}{4 \pi^{2}} \iint \frac{1}{r_{1}^{2} r_{2}^{2}}\left(\frac{l}{l_{0}}\right)^{D-E} e^{-2 i k\left(r_{1}^{2}-r_{2}^{2}\right)} d r_{1} d r_{2}= \\
& =\frac{A P_{t} \sum_{s}}{4 \pi^{2}} \int r_{1} d r_{1} d \theta_{1} \int r_{2} d r_{2} d \theta_{2} \frac{1}{r_{1}^{2} r_{2}^{2}}\left(\frac{l}{l_{0}}\right)^{D-E} e^{-2 i k\left(r_{1}^{2}-r_{2}^{2}\right)},
\end{aligned}
$$


where $l_{0}$ is the size of the fractal target, $l$ is comparable with the wavelength, $l<l_{0}$.

For the far zone $r_{1} \approx r_{2} \approx r$ and the flat target $E=2$. Then

$$
P_{s}=\frac{A P_{t} \sum_{s}}{4 \pi^{2}} \iint \frac{1}{r_{1}^{2} r_{2}^{2}}\left(\frac{|R|}{l_{0}}\right)^{D-2} e^{-2 i k\left(r_{1}^{2}-r_{2}^{2}\right)} d r_{1} d r_{2},
$$

where

$$
\begin{aligned}
& |R|=\left[r_{1}^{2}+R_{2}^{2}-2 r_{1} r_{2} \cos \left(\theta_{1}-\theta_{2}\right)\right]^{1 / 2} \approx \\
& \approx \sqrt{2} r \sqrt{1-\cos \left(\theta_{1}-\theta_{2}\right)} .
\end{aligned}
$$

Consequently, the power of the scattered signal with allowance for the results of [37]:

$$
\begin{aligned}
& P_{s}=\frac{A P_{t} \sum_{s}}{4 \pi^{2}} \int_{\theta_{01}}^{\theta_{01}^{\prime}} \int_{\theta_{02}}^{\prime}\left[1-\cos \left(\theta_{1}-\theta_{2}\right)^{(D-2) / 2}\right] d \theta_{1} d \theta_{2} \times \\
& \times \int_{r-(\Delta r / 2)}^{r+(\Delta r / 2)} d r_{1} \int_{r-(\Delta r / 2)}^{r+(\Delta r / 2)} \frac{2^{(D-2) / 2} r^{D-2}}{r^{2} l_{0}^{D-2}} e^{-2 i k(r-r)} d r_{2}= \\
& =\frac{2^{(D-2) / 2} A P_{t} \sum_{s}}{4 \pi^{2} r^{4-D} l_{0}^{D-2}} I_{r} I_{\theta} .
\end{aligned}
$$

In the expression (70), the integrals $I_{\mathrm{r}}$ and $I$ have the form

$$
\begin{aligned}
& I_{r}=\int_{r-(\Delta r / 2)}^{r+(\Delta r / 2)} d r_{1} \int_{r-(\Delta r / 2)}^{r+(\Delta r / 2)} e^{-2 i k\left(r_{1}-r_{2}\right)} d r_{2}=\frac{\sin ^{2}(k \Delta r)}{16 k^{2}}, \\
& I_{\theta}=\int_{\theta_{01}}^{\theta_{01}^{\prime}} d \theta_{1} \int_{\theta_{02}}^{\theta_{02}^{\prime}}\left[1-\cos \left(\theta_{1}-\theta_{2}\right)^{(D-2) / 2}\right] d \theta_{2},
\end{aligned}
$$

Consequently, on the basis of (70), we can conclude that for the fractal medium,

$$
P_{s} \propto \frac{1}{r^{4-D}} \text {. }
$$

The obtained result (73) coincides with the experimental data in Fig. 11 (rectilinear part of the graph) from [30].

For a $3 D$-medium, it is easy to obtain, taking into account the fact that in this case $E=3$, the following relation [37]:

$$
P_{s} \propto \frac{1}{r^{5-D}} \text {. }
$$

This result (74) agrees with the experimental data in Fig. 11 (the curvilinear part of the graph) from [30].

As noted in Ref. [37], the results show that the fractal dimension $D$ of the probed fractal medium or fractal target (such as the dynamic snow layer, etc., see also Refs $[12,13,26,27,37-40])$ can be estimated from the reflected radar signal.

\section{CONCLUSION}

The previously constructed modification of the theory of multiple scattering allowed us to include the value of the fractal dimension $D$ or the fractal signature $D(\mathbf{r}, t)$ of a disordered system.

Based on the modified Foldy-Twersky method for multiple scattering of waves in fractal discrete randomly inhomogeneous media [12, 13], the radiolocation equation for a purely fractal medium is analytically considered. It is shown that the fractal signature can be used to study the dependence of volume scattering on distance. Theoretical studies are consistent with previously published results of foreign authors.

Similarly, one can justify a solution for anisotropic disordered large fractal systems: cascades of fractals nested into each other, graphs from fractal chains, percolation systems, space debris, drones or small spacecraft (MCA) clusters, including mini and microclasses, dynamically synthesized space antenna groupings (cluster apertures), low-visible high-altitude pseudosatellites (HAPS) and their groupings, spatiallydistributed space systems (clusters) from small MKs solutions for emergency monitoring tasks, etc. [12, 13, 38-41].

The present study continues the author's cycle of studies on the justification of the application of the theory of fractals, physical scaling and fractional operators in radiophysics and radiolocation, initiated by the author for the first time in the USSR in the USSR Academy of Sciences in the late 70 s of the 20 th century.

\section{REFERENCES}

1. Foldy LL. The multiple scattering of waves. Phys. Rev., 1945, 67:107-119.

2. Twersky V. On the propagation in random media of discrete scatterers. Proc. Sympos. Appl. Math. (Am. Math. Soc., Providence, Rhode Island). 1964, 16:84-116.

3. Twersky V. Theory and Microwave Measurements of Higher Statistical Moments of Randomly Scattered Fields. In: Electromagnetic Scattering Proc. of the 
Interdisciplinary Conference held in June, 1965, at the University of Massachusetts at Amherst, Amherst, MA USA. Eds. R.L. Rowell, R.S. Stein. N.Y., Gordon and Breach, 1967, p. 579-696.

4. Rosenberg GW. Vektor-parametr Stoksa (Matrichnye metody ucheta polyarizatsii izlucheniya $\mathrm{v}$ priblizhenii luchevoy optiki) [Vector-Stokes parameter (Matrix methods for recording radiation polarization in the approximation of ray optics)]. UFN, 1955, 56 (1):77-110 (in Russ.).

5. Barabanenkov YuN. Mnogokratnoe rasseyanie voln na ansamble chastots i teoriya perenosa izlucheniya [Multiple scattering of waves by an ensemble of particles and the theory of radiation transfer]. UFN, 1975, 117 (1):49-78 (in Russ.).

6. Rytov SM, Kravtsov YuA, Tatarsky VI. Vvedenie v statisticheskuyu radiofiziku. Chast' 2. Sluchaynye polya Introduction to statistical radiophysics. Part II. Random fields]. Ed. SM Rytov. Moscow, Nauka Publ., 1978, 464 p.

7. Isimaru A. Propagation and scattering of waves in randomly inhomogeneous media. In 2 Vols. NY, Acad. Press, 1978.

8. Kravtsov YuA, Feizulin ZI, Vinogradov AG. Prokhozhdenie radiovoln cherez, atmosfery Zemli Passage of radio waves through the Earth's atmosphere]. Moscow, Radio i svyaz' Publ., 1983, 224 p.

9. Mishchenko Michael I, Travis Larry D, Lacis Andrew A. Multiple Scattering of Light by Particles: Radiative Transfer and Coherent Backscattering. N.Y., Cambridge University Press, 2006, 507 p.

10. Mandelbrot B. Fractals: Form, Chance, and Dimension. San Francisco, Freeman \& Co, 1977, $352 \mathrm{p}$.

11. Berry MV. Diffractals. J. Phys. A: Math. Gen., 1979, 12(6):781-797.

12. Potapov AA. Fraktaly v radiofizike $i$ radiolokatsii [Fractals in radiophysics and radar]. Moscow, Logos Publ., 2002, 664 p.

13. Potapov AA. Fraktaly v radiofizike $i$ radiolokatsii: topologiya vyborki [Fractals in Radiophysics and Radar: Topology of the sample]. Moscow, Universitetskaya kniga Publ., 2005, 848 p.
14. PotapovAA. Fraktalyikhaoskakosnova novykh propyvnykh tekhnologiy $\mathrm{V}$ sovremennykh radoisistemakh [Fractals and chaos as a basis for new breakthrough technologies in modern radio systems. Addition to the book: Kronover R. Fraktaly $i$ khaos $v$ dinamicheskikh sistemakh [Fractals and chaos in dynamic systems]. Trans. with English., Ed. E.E. Krenkel. Moscow, Tekhnosfera Publ., 2006, p. 374-479.

15. Alexander A. Potapov. Chaos Theory, Fractals and Scaling in the Radar: A Look from 2015. In: The Foundations of Chaos Revisited: From Poincaré to Recent Advancements. Switzerland, Basel, Springer Int. Publ., 2016, p. 195-218.

16. Potapov Alexander A. On the Indicatrixes of Waves Scattering from the Random Fractal Anisotropic Surface. In: Fractal Analysis Applications in Physics, Engineering and Technology. Ed. Fernando Brambila. Rijeka, InTech, 2017, p. 187-248.

17. Potapov Alexander A. Postulate "The Topology Maximum at the Energy Minimum" for Textural and Fractal-and-Scaling Processing of Multidimensional Super Weak Signals against a Background of Noises. In: Nonlinearity: Problems, Solutions and Applications, vol. 2. Ed. Ludmila A. Uvarova, Alexey B. Nadykto, and Anatoly V. Latyshev. New York, Nova Science Publ., 2017, p. 35-94.

18. Kitaev AE, Potapov AA, Rassadin AE. Vremennaya evolutsiya fraktal'nogo nachal'nogo usloviya pri roste poverkhnosti [Time evolution of the fractal initial condition with surface growth]. Trudy 2 Rossiyskobelarusskoy nauchno-tekhn. konf. : Elementnaya baza otechestvennoy radioelektroniki: importozameshchenie i primenenie" im. OV Loseva (Nizhny Novgorod, 17-19.11.2015). N. Novgorod, Lobachevsky NNSU Publ., 2015, p. 294-297 (in Russ.).

19. Kitaev AE, Potapov AA, Rassadin AE. Chislenno-analiticheskaya teoriya vozmushcheniy dlya modelirovaniya rosta fraktal'noy poverkhnosti [Numericalanalytic perturbation theory for modeling fractal surface growth]. Materialy 9 V seross. nauchnoy konf. "Matematicheskoe modelirovanie razvivayushcheysya ekonomiki, ekologii i tekhnologiy" 
EKOMOD-2016 (Kirov, 04-09.07.2016). Ed. I.G. Pospelova and A.V. Shatrova. Kirov, VyatGU Publ., 2016, p. 262-271 (in Russ.).

20. Kitaev AE, Potapov AA, Rassadin AE. Konkurentsiya gladkogo i fraktal'nogo profiley pri roste poverkhnosti tverdogo tela [Competition of smooth and fractal profiles with the growth of the surface of a solid]. Tez: dokl. 4 V seross. konf. i shkoly molodykh uchenykh $i$ spetsialistov "Fizicheskie i fiziko-khimicheskie osnovy ionnoy implantatsii". (Nizhny Novgorod, 24-27.10.2016). N. Novgorod, Lobachevsky NNSU Publ., 2016, p. 85-86 (in Russ.).

21. Kitaev AE, Potapov AA, Rassadin AE. Tochnoe reshenie dlya pervogo priblizheniya teorii vozmushcheniya uravneniya KardaraParizi-Zvanga [An exact solution for the first approximation of perturbation theory of the Kardar-Parisi-Zwang equation]. Tezisy dokl. Mezhb. konf.-shkoly "Shilnikov Seminar 2016" (N.Novgorod, Russia, 16-17.12.2016). Nizhny Novgorod, Lobachevsky NNGU Publ., 2016, p. 22 (in Russ.).

22. Kulikov DA, Potapov AA, Rassadin AE. Modelirovanie rosta fraktal'nykh struktur na poverkhnosti tverdykh tel s geksagonal'noy i kubicheskoy simmetriey [Modeling the growth of fractal structures on the surface of solids with hexagonal and cubic symmetry]. Materialy 10 Nauch.-prak.t. seminar" Aktual'nye problemy fiziki kondensirovannogo sostoyaniya [Actual problems of condensed matter physics]" $i$ vyezdnoy sessii Nauchnogo Soveta $R A N$ po fizike condensirovannogo sostoyaniya, posv. 110-letiyu H.I. Amirkhanova (Makhachkala, Russia, 06-09.06.2017). Makhachkala, Institute of Physics (Dagestan Scientific Center, Russian Academy of Sciences) Publ., 2017, p. 56-57 (in Russ.).

23. Kulikov DA, Potapov AA, Rassadin AE, Stepanov AV. Modelirovanie rosta fraktalov $\mathrm{s}$ tsilindricheskoy obrazuyushchey na poverkhnosti tverdogo tela [Modeling the growth of fractals with a cylindrical generatrix on the surface of a solid]. Tez: dokl. Mezhd. konf. "Skaniruyushchaya rondovaya mikroskopiya [Scanning Probe Microscopy]" (Ekaterinburg,
28-30.08.2017). Ekaterinburg, the Urals. feder. Univ., 2017, p. 281-282 (in Russ.).

24. Kulikov YES, Potapov AA, Rassadin AE. Perenos fraktal'nykh profiley i uravnenie Kardara-Parizi-Zvanga [The transfer of fractal profiles and the Kardar-Parisi-Zwang equation]. Trudy 10 Mezhd. konf. "Khaos $i$ struktury $v$ nelineynykh sistemakh [Chaos and Structures in Nonlinear Systems: Theory and Experiment]", posv. 75-letiyu prof. Z. Zhanabaeva (Kazakhstan, Almaty, 16-18.06.2017). Almaty, Al-Farabi Kazakh. natsion. un-t Publ., 2017, p. 82-86 (in Russ.).

25. Agalarov AM, Gadzhimuradov TA, Potapov AA, Rassadin AE. Effekty pervichnoy anizotropii pri roste poverkhnosti tverdogo tela s tsilindricheskoy obrazuyushchey [Effects of primary anisotropy with the growth of the surface of a solid body with a cylindrical generator]. Materialy 12 Mezhd. konf. "Fundamental'nye i prikladnye problemy matematiki $i$ informatiki [Fundamental and Applied Problems of Mathematics and Informatics]", posv. 85-letiyu prof. Alishaeva MG (Makhachkala, Russia, 1922.09.2017). Makhachkala, DGU Publ., 2017, p. 18-20 (in Russ.).

26. Kulikov DA, Potapov AA, Rassadin AE, Stepanov AV. Model for the growth of fractal solid state surface and the possibility of its verification by means of atomic force microscopy. IOP Conf. Ser.: Mater. Sci. Eng., 2017, 256(012026). doi: 10.1088/1757-899X.256.1.012026.

27. Potapov AA, Rassadin AE, Stepanov AV, Tronov AA. Nonlinear Dynamics of Fractals with Cylindrical Generatrix on Surface of Solid State. Proc. 14th Sino-Russia Symposium on Advanced Materials and Technologies. Ed. Mingxing Jia (Sanya, Hainan Province, China, 28.11-01.12.2017). Beijing, Metallurgical Industry Press, China, 2017, p. 491-493, http://www.cnmip.com.cn.

28. Mishchenko MI, Hovenier JW, Travis LD (eds.) Light Scattering by Nonspherical Particles: Theory, Measurements, and Applications. San Diego, Academic Press, 2000. 
29. Doviak R, Zrnich D. Doplerovskie radiolokatory $i$ meteorologicheskie nablyudeniya [Doppler radars and meteorological observations]. Leningrad, Gidrometeoizdat Publ., 1988, 512 p.

30. Rastogi PK, Scheucher KF. Range of scattering from a fractal medium: Simulation results. Radio Science, 1990, 25 (5):1057-1063.

31. Rottger J, Liu CH. Partial reflection and scattering of VHF radar signals from the clear atmosphere. Geophys. Res. Lett., 1978, 5:357-360.

32. Gage KS, Green JL. Evidence of specular reflections from monostatic VHF radar observations of the stratosphere. Radio Science 1978, 13:991-1001.

33. Woodman RF, Chu Y-H. Aspect sensitivity measurements of VHF backscatter made with the Chung-Li radar: Plausible mechanisms. Radio Science, 1989, 24:113-125.

34. Fritts DC, Rastogi PK. Convective and dynamical instabilities due to gravity wave motions in the lower and middle atmosphere: Theory and observations. Radio Science, 1985 , 20:1247-1277.

35. Rottger J. VHF radar measurements of smallscale and meso-scale dynamical processes in the middle atmosphere. Philos. Trans. R. Soc. London, Ser. A. 1987, 323:611-628.

36. Lovejoy S, Schertzer D. Scale invariance, symmetries and stochastic simulation of atmospheric phenomena. Bull. Am. Meteor. Soc., 1986, 67:21-32.

37. Zhen-song Wang, Bao-wei Lu. The scattering of electromagnetic waves in fractal media. Waves in Random Media, 1994, 4 (1):97-103.

38. Potapov AA. Theory of multiple scattering of electromagnetic waves in fractal discrete randomly inhomogeneous media. Part I. Waves in disordered fractal systems. Sat. doc. 24 Int. STC "Radiolocation, navigation, communications" (Voronezh, 18-20.04.2018). Voronezh, 2018 (in Russ.).

39. Potapov A.A. Waves in Large Disordered Anisotropic Fractal Systems, in Clusters of Small-Size Space Vehicles, in Synthesized Space Antenna Aggregations - Cluster Apertures, and in Radar. Book of Abstracts Int. Conf.School "Shilnikov WorkShop 2017" (Nizhni
Novgorod, 15-16.12, 2017). N. Novgorod: Lobachevsky State University, 2017, p. 55-56.

40. Potapov A.A. Fractal and topological sustainable methods of overcoming expected uncertainty in the radiolocation of lowcontrast targets and in the processing of weak multi-dimensional signals on the background of high-intensity noise: A new direction in the statistical decision theory. Journal of Physics: Conference Series. Institute of Physics (United Kingdom) Publ., 2017, 918:012015; doi: 10.1088/1742-6596.918.1.012015.

41. Potapov AA, German VA. Book of Abstr. 11th Chaotic Modeling and Simulation Intern. Conf. (Rome, Italy: 5-8.06.2018). Rome, ISAST, 2018. 